\title{
Lindelöf indestructibility, topological games and selection principles
}

by

\author{
Marion Scheepers (Boise, ID) and Franklin D. Tall (Toronto)
}

\begin{abstract}
Arhangel'skii proved that if a first countable Hausdorff space is Lindelöf, then its cardinality is at most $2^{\aleph_{0}}$. Such a clean upper bound for Lindelöf spaces in the larger class of spaces whose points are $\mathrm{G}_{\delta}$ has been more elusive. In this paper we continue the agenda started by the second author, [Topology Appl. 63 (1995)], of considering the cardinality problem for spaces satisfying stronger versions of the Lindelöf property. Infinite games and selection principles, especially the Rothberger property, are essential tools in our investigations.
\end{abstract}

A topological space is Lindelöf if each open cover contains a countable subset that covers the space. Aleksandrov asked if in the class of first countable Hausdorff spaces, every Lindelöf space has cardinality at most $2^{\aleph_{0}}$. Arhangel'skii [1] proved that the answer is "yes". This focuses attention on the larger class of spaces in which "points are $\mathrm{G}_{\delta}$ " - i.e., the class of topological spaces in which each point is an intersection of countably many open sets. Such spaces are $T_{1}$ but not necessarily Hausdorff. Arhangel'skii showed that in the class of spaces with points $\mathrm{G}_{\delta}$ each Lindelöf space has cardinality less than the least measurable cardinal. Juhász [26] showed that this bound is sharp: There are such Lindelöf spaces of arbitrarily large cardinality below the least measurable cardinal. Juhász's examples are not Hausdorff spaces and the cardinality of the underlying spaces has countable cofinality. Shelah [45] showed that no Lindelöf space with points $\mathrm{G}_{\delta}$ can be of weakly compact cardinality. Gorelic [19] showed that it is relatively consistent that the Continuum Hypothesis $(\mathrm{CH})$ holds, that $2^{\aleph_{1}}$ is arbitrarily large, and there is a zero-dimensional $\mathrm{T}_{1}$ Lindelöf space with points $\mathrm{G}_{\delta}$ and of cardinality $2^{\aleph_{1}}$. This improved earlier results of Shelah [45], Shelah-Stanley [46] and

2010 Mathematics Subject Classification: 54A25, 54A35, 54D20.

Key words and phrases: indestructibly Lindelöf, infinite game, Rothberger space, GerlitsNagy space, Menger property, Hurewicz property, measurable cardinal, weakly compact cardinal, real-valued measurable cardinal, Cohen reals, random reals. 
Velleman [59] which showed that either by countably closed forcing, or by assuming $\mathrm{V}=\mathrm{L}$, one could obtain such a space of cardinality $\aleph_{2}$, consistent with $\mathrm{CH}$. Little else is known about cardinality and the Lindelöf property in the class of spaces with points $\mathrm{G}_{\delta}\left({ }^{1}\right)$, It is not even known (absolutely or consistently) if all such $T_{2}$ spaces have cardinality $\leq 2^{\aleph_{1}}$.

This cardinality problem is of interest also for stronger versions of the Lindelöf property. An interesting strengthening of the Lindelöf property is identified and studied in [51]: Call a Lindelöf space indestructibly Lindelöf if in any generic extension by a countably closed partially ordered set the space is still Lindelöf. [51, Theorem 19] shows that Juhász's examples are destructible. And [51, Theorem 4] shows, assuming large cardinals, that it is consistent that in the class of spaces with points $\mathrm{G}_{\delta}$, each indestructibly Lindelöf space has cardinality at most $2^{\aleph_{0}}$. It is also shown in [51] (in the paragraph following Theorem 18) that Gorelic's space is indestructibly Lindelöf. Thus, the statement that indestructibly Lindelöf spaces with points $\mathrm{G}_{\delta}$ are of cardinality $\leq 2^{\aleph_{0}}$ is (modulo large cardinals) independent. This independence can also be obtained in another way if the separation hypothesis is weakened: A. Dow [13] showed that adding $\aleph_{1}$ Cohen reals converts every ground model Lindelöf space to an indestructibly Lindelöf space in the generic extension. This gives the consistency of the existence of non- $T_{2}$ indestructibly Lindelöf spaces with points $\mathrm{G}_{\delta}$ of arbitrarily large cardinality below the first measurable cardinal: Juhász's spaces from the ground model retain their cardinality but acquire Lindelöf indestructibility, and measurable cardinals from the ground model remain measurable.

[51, Theorem 3] gives a combinatorial characterization of indestructibly Lindelöf. In Section 1, starting from this combinatorial characterization, we show how to characterize indestructibly Lindelöf game-theoretically (Theorem 11) and examine the determinacy of this game. This analysis leads us to two natural strengthenings of indestructibly Lindelöf. For one of these strengthenings, all spaces with points $\mathrm{G}_{\delta}$ and this strengthening have cardinality $\leq 2^{\aleph_{0}}$ (Theorem 2). The classical selection property introduced by Rothberger is the other natural strengthening (Corollary 10). These topics are illustrated with some examples collected in Section 4.

In Section 2 we focus on the Rothberger property. In [15, using the technique of $n$-dowments, it was shown that a variety of non-covering and non-generalized-metric properties were preserved by Cohen reals. Since these arguments mimic measure-theoretic ones, the same preservation arguments

$\left({ }^{1}\right)$ R. Knight has published a paper 29 ] claiming that V $=$ L implies there are Lindelöf spaces with points $\mathrm{G}_{\delta}$ of size $\aleph_{n}$, for each $n \leq \omega$ (see Theorems 3.6.1, 3.6.2 and 3.6.3 of 29]). Questions have been raised as to whether the proof is correct. In [51] a similar result was attributed to C. Morgan, but that proof turned out to be incorrect. 
work for adding random reals. This work was extended by [20] and by [23]. It turns out that covering properties are also often preserved. From this previous work, it was not at all evident that there would be some covering or non-covering properties which would be preserved by one of these kinds of extensions, but not the other. Here we shall show that while "nonRothberger" is not preserved by Cohen extensions (of uncountable cardinality) it is preserved by random real extensions. Specifically: We show that forcing with $\kappa$ Cohen reals ( $\kappa$ uncountable) converts all ground model Lindelöf spaces to spaces with the Rothberger property (Theorem 11). This improves the above-mentioned result of A. Dow. We show that forcing with $\kappa$ random reals preserves the Rothberger property, and for a large class of spaces, forcing with $\kappa$ random reals preserves the non-Rothberger property (Theorems 15 and 17). We also show that Rothberger spaces not only retain the Lindelöf property under countably closed forcing (since they are indestructibly Lindelöf) but in fact they retain the Rothberger property (Theorem 21).

These three results lead to several illuminating pieces of information about the (insufficiently studied) Rothberger property in general spaces: Every Rothberger space with points $\mathrm{G}_{\delta}$ has cardinality no larger than the least real-valued measurable cardinal (Theorem 24). If it is consistent that there is a measurable cardinal, then it is consistent that all Rothberger spaces with points $G_{\delta}$ have cardinality at most $2^{\aleph_{0}}$. (Even $T_{3}$ ) Rothberger spaces of cardinality larger than $\aleph_{1}$ need not include a Lindelöf subspace of cardinality $\aleph_{1}$. However it is consistent, assuming the consistency of the existence of a measurable cardinal, that Rothberger $T_{2}$ spaces of character less than or equal to $\aleph_{1}$ include Rothberger subspaces of size at most $\aleph_{1}$ (Theorem 35). We also find a new forcing proof for Shelah's result that there is no points $G_{\delta}$ Lindelöf topology on a set of weakly compact cardinality (Proposition 34).

In Section 3 we examine a covering property introduced by Gerlits and Nagy [18]. The Gerlits-Nagy property is closely related to the Rothberger property. Indeed, for regular spaces it is undecidable by ZFC whether these two properties are different. Study of the Gerlits-Nagy property requires considering the preservation, by forcing, of selection properties introduced by Hurewicz. One of these selection properties is called the Menger property, and the other the Hurewicz property. Unlike the Menger property and the Rothberger property, both Cohen real and random real forcing preserve being not Hurewicz (Theorems 37 and 43 . While Cohen real forcing does not preserve the Hurewicz property in general, random real forcing does (Theorem 44). We also found a class of separable metrizable spaces (which include Sierpiński sets) for which Cohen forcing does preserve the Hurewicz property (Theorem 40). Thus it is consistent that the square of a set of real 
numbers with the Gerlits-Nagy property need not have the Gerlits-Nagy property (Corollary 48). This solves Problem 6.6 of [56].

In Section 4 we remark on a few examples in light of our results. We show that Gorelic's example has both the Rothberger and Hurewicz properties while Juhász's examples have the Hurewicz property but not the Rothberger property.

1. Indestructibly Lindelöf and an infinite game. Let $\mathcal{A}$ and $\mathcal{B}$ be collections of sets and let $\alpha$ be an ordinal number. Then the game $\mathrm{G}_{1}^{\alpha}(\mathcal{A}, \mathcal{B})$ between players ONE and TWO is played as follows: They play an inning for each $\beta<\alpha$. In the $\beta$ th inning ONE first chooses some $\mathcal{U}_{\beta} \in \mathcal{A}$; TWO responds by choosing some set $T_{\beta} \in \mathcal{U}_{\beta}$. A play

$$
\mathcal{U}_{0}, T_{0}, \ldots, \mathcal{U}_{\beta}, T_{\beta}, \ldots: \beta<\alpha
$$

is won by TWO if $\left\{T_{\beta}: \beta<\alpha\right\}$ is a member of $\mathcal{B}$; else, ONE wins.

Let $\mathcal{O}$ denote the set of all open covers of a space $X$. In [17] Galvin introduced the version $\mathrm{G}_{1}^{\omega}(\mathcal{O}, \mathcal{O})$ of the above game.

A collection $\left(U_{f}: f \in \bigcup_{\alpha<\omega_{1}}{ }^{\alpha} \omega\right)$ of open subsets of $X$ is said to be a covering tree if for each $\alpha<\omega_{1}$, for each $f \in{ }^{\alpha} \omega$, the set $\left\{U_{f \frown\{(\alpha, n)\}}\right.$ : $n<\omega\}$ is a cover of $X$. In Theorem 3 of [51] it is proved that a space $X$ is indestructibly Lindelöf if, and only if, it is Lindelöf, and for each covering tree $\left(U_{f}: f \in \bigcup_{\alpha<\omega_{1}}{ }^{\alpha} \omega\right)$ of $X$ there is an $f \in \bigcup_{\alpha<\omega_{1}}{ }^{\alpha} \omega$ such that the set $\left\{U_{f\left\lceil_{\beta}\right.}: \beta<\operatorname{dom}(f)\right\}$ is a cover of $X$.

TheOREM 1. For a Lindelöf space $X$ the following are equivalent:

1. $X$ is indestructibly Lindelöf.

2. ONE has no winning strategy in $\mathrm{G}_{1}^{\omega_{1}}(\mathcal{O}, \mathcal{O})$.

Proof. To see that $1 \Rightarrow 2$, observe that if $F$ is a strategy for player ONE then there is a natural covering tree associated with $F$ : We may assume, since $X$ is Lindelöf, that in each inning $F$ calls on ONE to play a countable open cover of $X$. Thus enumerate ONE's first move $F(\emptyset)$ as $\left(U_{(0, n)}: n<\omega\right)$. When TWO chooses $U_{\left(0, n_{0}\right)} \in F(\emptyset)$, ONE's response $F\left(U_{\left(0, n_{0}\right)}\right)$ may be enumerated as $\left(U_{\left\{\left(0, n_{0}\right),(1, n)\right\}}: n<\omega\right)$. When TWO chooses $U_{\left\{\left(0, n_{0}\right),\left(1, n_{1}\right)\right\}} \in$ $F\left(U_{\left\{\left(0, n_{0}\right)\right\}}\right)$, ONE's response $F\left(U_{\left(0, n_{0}\right)}, U_{\left.\left\{\left(0, n_{0}\right),\left(1, n_{1}\right)\right)\right\}}\right)$ is enumerated as $\left(U_{\left.\left\{\left(0, n_{0}\right),\left(1, n_{1}\right),(2, n)\right)\right\}}: n<\omega\right)$, and so on.

This produces the covering tree $\left(U_{f}: f \in \bigcup_{\alpha<\omega_{1}}{ }^{\alpha} \omega\right)$ of $X$, associated as above with ONE's strategy $F$. Now apply the fact that $X$ is indestructibly Lindelöf to find an $\alpha<\omega_{1}$ and an $f \in{ }^{\alpha} \omega$ such that $\left\{U_{f\left\lceil_{\beta}\right.}: \beta<\alpha\right\}$ is a cover of $X$. TWO wins the play

$$
F(\emptyset), U_{f\left\lceil_{1}\right.}, F\left(U_{f\left\lceil_{1}\right.}\right), \ldots, F\left(U_{f\left\lceil_{\beta}\right.}: \beta<\gamma\right), U_{f\left\lceil_{\gamma+1}\right.}, \ldots,
$$

demonstrating that $F$ is not a winning strategy for ONE. 
To see that $2 \Rightarrow 1$, observe that a covering tree rather directly defines a strategy of ONE in the game $\mathrm{G}_{1}^{\omega_{1}}(\mathcal{O}, \mathcal{O})$, and that a winning play of TWO against this strategy witnesses the indestructibility of $X$ for this covering tree.

Theorem 1 suggests a number of at least formal strengthenings of the notion of indestructibly Lindelöf. We consider specifically the following two: One strengthening is to require that TWO has a winning strategy in $G_{1}^{\omega_{1}}(\mathcal{O}, \mathcal{O})$; the second is to require that ONE has no winning strategy already in the shorter game $\mathrm{G}_{1}^{\omega}(\mathcal{O}, \mathcal{O})$.

When TWO has a winning strategy. If TWO has a winning strategy in $G_{1}^{\omega_{1}}(\mathcal{O}, \mathcal{O})$ then every open cover of the space contains a subcover of cardinality at most $\aleph_{1}$. In Theorem 2 we do not assume that the space is Lindelöf and use an idea of F. Galvin [17] in the proof.

TheOREM 2. Let $X$ be a space in which each point is a $\mathrm{G}_{\delta}$. If TWO has a winning strategy in the game $\mathrm{G}_{1}^{\omega_{1}}(\mathcal{O}, \mathcal{O})$ then $|X| \leq 2^{\aleph_{0}}$.

Proof. Since each point of $X$ is a $\mathrm{G}_{\delta}$, fix for each $x \in X$ a sequence $\left(U_{n}(x): n<\omega\right)$ of open sets with $\{x\}=\bigcap_{n<\omega} U_{n}(x)$. Let $F$ be a strategy for TWO. Let $\alpha<\omega_{1}$ as well as a sequence $\left(\mathcal{U}_{\beta}: \beta<\alpha\right)$ of open covers of $X$ be given.

Claim 1. There is an $x \in X$ such that for each open set $U \subseteq X$ with $x \in U$, there is an open cover $\mathcal{U}$ of $X$ with

$$
U=F\left(\left(\mathcal{U}_{\beta}: \beta<\alpha\right) \frown(\mathcal{U})\right) .
$$

Proof of Claim 1. If not, choose for each $x \in X$ an open set $U_{x} \subseteq X$ such that $x \in U_{x}$ and $U_{x}$ is a counterexample to the above statement. Then $\mathcal{U}=\left\{U_{x}: x \in X\right\}$ is an open cover of $X$, and $F\left(\left(\mathcal{U}_{\beta}: \beta<\alpha\right) \frown(\mathcal{U})\right) \in \mathcal{U}$, contradicting the definition of members of $\mathcal{U}$.

By the claim choose $x_{\emptyset} \in X$ so that there is for each open set $U$ with $x_{\emptyset} \in U$ an open cover $\mathcal{U}$ of $X$ with $U=F(\mathcal{U})$. For each $n$ choose an open cover $\mathcal{U}_{\{(0, n)\}}$ of $X$ with $U_{n}\left(x_{\emptyset}\right)=F\left(\mathcal{U}_{\{(0, n)\}}\right)$.

For each $n<\omega$ choose $x_{\langle n\rangle}$ for the open cover $\mathcal{U}_{\{(0, n)\}}$ as in the claim. Then, for each $n_{0}$ and each $n$ choose an open cover $\mathcal{U}_{\left\{\left(0, n_{0}\right),(1, n)\right\}}$ of $X$ such that

$$
U_{n}\left(x_{\left\langle n_{0}\right\rangle}\right)=F\left(\mathcal{U}_{\left\{\left(0, n_{0}\right)\right\}}, \mathcal{U}_{\left\{\left(0, n_{0}\right),(1, n)\right\}}\right) .
$$

Then for each $n_{0}, n_{1}<\omega$ choose $x_{\left\langle n_{0}, n_{1}\right\rangle} \in X$ as in the claim for the sequence $\left(\mathcal{U}_{\left\{\left(0, n_{0}\right)\right\}}, \mathcal{U}_{\left\{\left(0, n_{0}\right),(1, n)\right\}}\right)$ of open covers. For each $n<\omega$ choose an open cover $\mathcal{U}_{\left\{\left(0, n_{0}\right),\left(1, n_{1}\right),(2, n)\right\}}$ of $X$ such that

$$
U_{n}\left(x_{\left\langle n_{0}, n_{1}\right\rangle}\right)=F\left(\mathcal{U}_{\left\{\left(0, n_{0}\right)\right\}}, \mathcal{U}_{\left\{\left(0, n_{0}\right),(1, n)\right\}}, \mathcal{U}_{\left\{\left(0, n_{0}\right),\left(1, n_{1}\right),(2, n)\right\}}\right),
$$


and so on. In general, let $\alpha<\omega_{1}$ be given and assume we have selected for each $f \in \bigcup_{\beta<\alpha}{ }^{\beta} \omega$ points $x_{f} \in X$ and open covers $\mathcal{U}_{f}$ of $X$ such that for all $\beta<\alpha$ and for $f \in{ }^{\beta} \omega$ and $n<\omega$,

$$
U_{n}\left(x_{f}\right)=F\left(\left(\mathcal{U}_{f\left\lceil_{\gamma}\right.}: \gamma<\beta\right) \frown\left(\mathcal{U}_{f \cup\{(\beta, n)\}}\right)\right) .
$$

Consider $f \in{ }^{\alpha} \omega$. Applying Claim 1 to the sequence $\left(\mathcal{U}_{f\left\lceil_{\beta}\right.}: \beta<\alpha\right)$ of open covers, choose an $x_{f} \in X$ as in Claim 1. Then for each $n<\omega$ choose an open cover $\mathcal{U}_{f \cup\{(\alpha, n)\}}$ of $X$ with

$$
\left.U_{n}\left(x_{f}\right)=F\left(\mathcal{U}_{f{ }_{\beta}}: \beta<\alpha\right) \frown\left(\mathcal{U}_{f \cup\{(\alpha, n)\}}\right)\right) .
$$

This specifies how to recursively choose for each $f \in \bigcup_{\alpha<\omega_{1}}{ }^{\alpha} \omega$ a point $x_{f} \in X$ and an open cover $\mathcal{U}_{f}$ such that for each such $f$ and each $n<\omega$, if $f \in{ }^{\gamma} \omega$, then

$$
\left.U_{n}\left(x_{f}\right)=F\left(\mathcal{U}_{f\left\lceil_{\beta}\right.}: \beta<\gamma\right) \frown\left(\mathcal{U}_{f \cup\{(\gamma, n)\}}\right)\right) .
$$

Note that $\left\{x_{f}: f \in \bigcup_{\alpha<\omega_{1}}{ }^{\alpha} \omega\right\}$ has cardinality at most $2^{\aleph_{0}}$.

Claim 2. $X=\left\{x_{f}: f \in \bigcup_{\alpha<\omega_{1}}{ }^{\alpha} \omega\right\}$.

Proof of Claim 2. For if not, choose $x \in X \backslash\left\{x_{f}: f \in \bigcup_{\alpha<\omega_{1}}{ }^{\alpha} \omega\right\}$. Choose $n_{0}$ with $x \notin U_{n_{0}}\left(x_{\emptyset}\right)$. ONE plays $\mathcal{U}_{\left\{\left(0, n_{0}\right)\right\}}$ and TWO responds using $F$. Choose $n_{1}$ with $x \notin U_{n_{1}}\left(x_{\left\langle n_{0}\right\rangle}\right)$. Next ONE plays $\mathcal{U}_{\left\{\left(0, n_{0}\right),\left(1, n_{1}\right)\right\}}$ and TWO responds using $F$, and so on. In this way we build an $F$-play which is lost by TWO since TWO never covers the point $x$. This contradicts the fact that $F$ is a winning strategy for TWO.

A result of [12] can be formulated as follows.

Theorem 3 (Daniels-Gruenhage). If $X$ is hereditarily Lindelöf then TWO has a winning strategy in $\mathrm{G}_{1}^{\omega_{1}}(\mathcal{O}, \mathcal{O})$.

Combining Theorems 2 and 3 gives a new proof for an old result of de Groot:

COROLlary 4. If $X$ is hereditarily Lindelöf and each point is a $\mathrm{G}_{\delta}$-set, then $|X| \leq 2^{\aleph_{0}}$. Thus if $X$ is hereditarily Lindelöf and $\mathrm{T}_{2}$, then $|X| \leq 2^{\aleph_{0}}$.

Determinacy of the game $\mathrm{G}_{1}^{\omega_{1}}(\mathcal{O}, \mathcal{O})$. If a topological space $X$ has cardinality $\leq \aleph_{1}$, then TWO has a winning strategy in $G_{1}^{\omega_{1}}(\mathcal{O}, \mathcal{O})$ on that space. Thus:

Corollary 5. Assume the Continuum Hypothesis. For a Lindelöf space $X$ with points $\mathrm{G}_{\delta}$ the following are equivalent:

1. TWO has a winning strategy in $\mathrm{G}_{1}^{\omega_{1}}(\mathcal{O}, \mathcal{O})$.

2. $|X| \leq \aleph_{1}$.

An infinite two-player game in which neither player has a winning strategy is said to be undetermined. In the class of Lindelöf spaces with points 
$\mathrm{G}_{\delta}$ the determinacy of the game $\mathrm{G}_{1}^{\omega_{1}}(\mathcal{O}, \mathcal{O})$ is (modulo large cardinals) independent of ZFC. This can be seen as follows:

Theorem 6 (Gorelic, [19]). For any cardinal number $\aleph_{\alpha}$ it is consistent, relative to the consistency of ZFC, that $2^{\aleph_{0}}=\aleph_{1}$ and there is a zerodimensional Lindelöf space $X$ with points $\mathrm{G}_{\delta}$ and $|X|=2^{\aleph_{1}} \geq \aleph_{\alpha}$.

Shelah's earlier proof of consistency of existence of such a space in a model where $\aleph_{1}=2^{\aleph_{0}}<2^{\aleph_{1}}=\aleph_{2}$ finally appeared in [45]. Note that such a space is necessarily $T_{3}$ : points $G_{\delta}$ implies $T_{1}$, and $T_{1}$ plus zero-dimensional implies $\mathbf{T}_{3}$. Tall showed in [51] that both Shelah's and Gorelic's examples are indestructibly Lindelöf. Theorems 1 and 2 imply that the game $\mathrm{G}_{1}^{\omega_{1}}(\mathcal{O}, \mathcal{O})$ is undetermined on these two spaces.

In Theorem 4 of [51] it is proved consistent, relative to the consistency of the existence of a supercompact cardinal, that the indestructibly Lindelöf spaces with points $\mathrm{G}_{\delta}$ are of small cardinality:

ThEOREM 7 (Tall). If it is consistent that there is a supercompact cardinal, then it is consistent that the Continuum Hypothesis holds and every indestructibly Lindelöf space with points $\mathrm{G}_{\delta}$ is of cardinality $\leq 2^{\aleph_{0}}$.

This yields the following determinacy result:

COROLlARY 8. If it is consistent that there is a supercompact cardinal, then it is consistent that the game $\mathrm{G}_{1}^{\omega_{1}}(\mathcal{O}, \mathcal{O})$ is determined on all Lindelöf spaces with points $\mathrm{G}_{\delta}$.

Proof. Consider a model of Theorem 7. CH holds in this model. Consider in this model a Lindelöf space $X$ with points $\mathrm{G}_{\delta}$. If $|X| \leq \aleph_{1}$ then by Corollary 5. TWO has a winning strategy in $\mathrm{G}_{1}^{\omega_{1}}(\mathcal{O}, \mathcal{O})$. If $|X|>\aleph_{1}$, then by Theorem 4 of [51], $X$ is destructible. By Theorem 1, ONE has a winning strategy in $\mathrm{G}_{1}^{\omega_{1}}(\mathcal{O}, \mathcal{O})$.

When ONE does not win the shorter game $G_{1}^{\omega}(\mathcal{O}, \mathcal{O})$. There is a natural selection principle associated with the game $\mathrm{G}_{1}^{\alpha}(\mathcal{A}, \mathcal{B})$ : The symbol $\mathrm{S}_{1}^{\alpha}(\mathcal{A}, \mathcal{B})$ denotes the statement:

For each sequence $\left(A_{\gamma}: \gamma<\alpha\right)$ of elements of $\mathcal{A}$, there is a sequence $\left(B_{\gamma}: \gamma<\alpha\right)$ such that $B_{\gamma} \in A_{\gamma}$ for each $\gamma$, and $\left\{B_{\gamma}: \gamma<\alpha\right\} \in \mathcal{B}$.

If $\kappa$ is an initial ordinal with the same cardinality as $\alpha$, then $S_{1}^{\kappa}(\mathcal{A}, \mathcal{B})$ holds if, and only if, $\mathrm{S}_{1}^{\alpha}(\mathcal{A}, \mathcal{B})$ holds. Thus, we consider $\mathrm{S}_{1}^{\alpha}(\mathcal{A}, \mathcal{B})$ only for initial ordinals.

If ONE does not have a winning strategy in $\mathrm{G}_{1}^{\alpha}(\mathcal{A}, \mathcal{B})$, then $\mathrm{S}_{1}^{\alpha}(\mathcal{A}, \mathcal{B})$ holds. Thus, if $X$ is indestructibly Lindelöf, then it has the property $S_{1}^{\omega_{1}}(\mathcal{O}, \mathcal{O})$. This observation gives an easy proof that the compact space ${ }^{\omega_{1}} 2$ is not indestructibly Lindelöf: For $\alpha<\omega_{1}$ put $U_{i}^{\alpha}=\left\{f \in \omega_{1} 2: f(\alpha)=i\right\}$. 
Then each $\mathcal{U}_{\alpha}:=\left\{U_{0}^{\alpha}, U_{1}^{\alpha}\right\}$ is an open cover of $\omega_{1} 2$ and the sequence $\left(\mathcal{U}_{\alpha}: \alpha<\omega_{1}\right)$ witnesses that $S_{1}^{\omega_{1}}(\mathcal{O}, \mathcal{O})$ fails for this space.

Problem 1. When does $\mathrm{S}_{1}^{\omega_{1}}(\mathcal{O}, \mathcal{O})$ imply that $O N E$ has no winning strategy in $\mathrm{G}_{1}^{\omega_{1}}(\mathcal{O}, \mathcal{O})$ ?

Rothberger introduced the selection property $\mathrm{S}_{1}^{\omega}(\mathcal{O}, \mathcal{O})$ in [41]. Spaces with the property $S_{1}^{\omega}(\mathcal{O}, \mathcal{O})$ are said to have the Rothberger property, and are called Rothberger spaces. We shall use the notation $S_{1}^{\omega}(\mathcal{O}, \mathcal{O})$ and the terminology "Rothberger space". Note that the Rothberger property is inherited by closed subspaces and preserved by countable unions and continuous images. We shall often use the following observation about spaces $X$ with the property $S_{1}^{\omega}(\mathcal{O}, \mathcal{O})$ : for each sequence $\left(\mathcal{U}_{n}: n<\omega\right)$ of open covers of $X$ there is a sequence $\left(U_{n}: n<\omega\right)$ such that for each $n, U_{n} \in \mathcal{U}_{n}$, and for each $x \in X$ the set $\left\{n: x \in U_{n}\right\}$ is infinite. This follows by partitioning $\omega$ into countably many disjoint infinite sets $\left(S_{n}, n<\omega\right)$, and then applying $\mathrm{S}_{1}^{\omega}(\mathcal{O}, \mathcal{O})$ to each of the sequences $\left(\mathcal{U}_{n}: n \in S_{m}\right), m<\omega$, of open covers of $X$.

Theorem 9 (Pawlikowski, [38]). A topological space $X$ has property $\mathrm{S}_{1}^{\omega}(\mathcal{O}, \mathcal{O})$ if, and only if, ONE has no winning strategy in the game $\mathrm{G}_{1}^{\omega}(\mathcal{O}, \mathcal{O})$.

If ONE has no winning strategy in $\mathrm{G}_{1}^{\omega}(\mathcal{O}, \mathcal{O})$, then ONE has no winning strategy in $\mathrm{G}_{1}^{\omega_{1}}(\mathcal{O}, \mathcal{O})$. It follows that:

COROLlaRY 10. Rothberger spaces are indestructibly Lindelöf.

Unlike the case with the game $G_{1}^{\omega_{1}}(\mathcal{O}, \mathcal{O})$ (see Corollary 8), the game $\mathrm{G}_{1}^{\omega}(\mathcal{O}, \mathcal{O})$ is provably undetermined in the class of points $\mathrm{G}_{\delta}$ Lindelöf spaces: J. T. Moore's L-space (Section 4, Example 2) and Todorčević's stationary Aronszajn lines (Section 4, Example 6). These are uncountable Rothberger spaces with points $\mathrm{G}_{\delta}$. Since these spaces are Rothberger spaces, Pawlikowski's Theorem implies that ONE has no winning strategy in $G_{1}^{\omega}(\mathcal{O}, \mathcal{O})$. In [17] Galvin proved that if TWO has a winning strategy in $G_{1}^{\omega}(\mathcal{O}, \mathcal{O})$ in a points $\mathrm{G}_{\delta}$ Lindelöf space $X$, then $X$ is countable. Since Moore's and Todorčević's spaces are uncountable, also TWO has no winning strategy in $\mathrm{G}_{1}^{\omega}(\mathcal{O}, \mathcal{O})$.

Examples 2 and 3 are spaces of cardinality $\aleph_{1}$, and the spaces of Example 6 can also be taken to be of cardinality $\aleph_{1}$. Thus, for each of these spaces TWO has a winning strategy in $G_{1}^{\omega_{1}}(\mathcal{O}, \mathcal{O})$. In Example 3 of Section 4, we examine Gorelic's space more closely and show that it is a Rothberger space, whence ONE has no winning strategy in $G_{1}^{\omega}(\mathcal{O}, \mathcal{O})$ and TWO has no winning strategy in $G_{1}^{\omega_{1}}(\mathcal{O}, \mathcal{O})$ on this example.

As will be seen later, in the class of metrizable spaces the determinacy of the game $\mathrm{G}_{1}^{\omega}(\mathcal{O}, \mathcal{O})$ is independent of ZFC. 
2. The Rothberger property. The Rothberger property arose in connection with the classical Borel Conjecture. E. Borel [8] defined a subset $X$ of the real line $\mathbb{R}$ to be strong measure zero if for each sequence $\left(\epsilon_{n}: n \in \mathbb{N}\right)$ of positive real numbers there is a sequence $\left(I_{n}: n \in \mathbb{N}\right)$ of open intervals such that for each $n$ length $\left(I_{n}\right) \leq \epsilon_{n}$, and $X \subseteq \bigcup_{n \in \mathbb{N}} I_{n}$. Borel conjectured that all strong measure zero sets of reals are countable. The statement that strong measure zero sets of real numbers are countable sets is known as the Borel Conjecture. Rothberger [41] showed that for metrizable spaces the Rothberger property implies strong measure zero. Indeed, for metrizable spaces the Rothberger property is equivalent to having strong measure zero in all equivalent metrics [16].

Rothberger spaces have been rarely considered outside the metric context. Indeed, to our knowledge, cardinality restrictions on Rothberger spaces have received no prior attention outside the metric context.

Rothberger spaces and Cohen reals. For an infinite cardinal number $\kappa$, let $\mathrm{Fn}(\kappa, 2)$ denote the set of finite binary functions with domain a finite subset of $\kappa$. For $f$ and $g$ elements of $\operatorname{Fn}(\kappa, 2)$ write $f<g$ to denote that $f$ extends $g$. Thus, $(\mathrm{Fn}(\kappa, 2),<)$ is one of several partially ordered sets for adding $\kappa$ Cohen reals. $\mathrm{Fn}(\kappa, \omega)$, the set of functions with domain a finite subset of $\kappa$ and values in $\omega$, and with the same order as above, leads to the same generic extension. We shall use the symbol $\mathbb{P}(\kappa)$ to denote either of these posets for adding $\kappa$ Cohen reals.

TheOREM 11. Let $\kappa$ be an uncountable cardinal number. If $X$ is a Lindelöf space, then $\mathbf{1}_{\mathbb{P}(\kappa)} \|-$ "X has property $\mathrm{S}_{1}^{\omega}(\mathcal{O}, \mathcal{O})$ ".

Proof. Let $X$ be a Lindelöf space. Let $\kappa$ be an uncountable cardinal. Everywhere below when we write $\mathbb{P}(\kappa)$, we may consider this forcing to be expressed as $\mathbb{P}\left(\kappa+\omega_{1}\right)$. It is known (see [13] or [20]) that $\mathbf{1}_{\mathbb{P}(\kappa) \|-} \|$ " $\check{X}$ is Lindelöf". Consider a $\mathbb{P}(\kappa)$-name for a sequence of open covers of $X$, say $\left(\dot{\mathcal{U}}_{n}: n \in \mathbb{N}\right)$. Since the ground-model topology of $X$ is a basis for the topology in the generic extension, and since $X$ is Lindelöf, we may assume that

$\mathbf{1}_{\mathbb{P}(\kappa)} \|-$ "Each $\dot{\mathcal{U}}_{n}$ is a countable cover by ground-model open sets".

Indeed, for each $n$ and $m$ we can choose a maximal antichain $A_{m}^{n}$ of $\mathbb{P}(\kappa)$ such that the $m$ th element of $\dot{\mathcal{U}}_{n}$ is decided by this antichain. Considering $\mathbb{P}(\kappa)$ as $\mathbb{P}\left(\kappa+\omega_{1}\right)$, and since each member of each $A_{m}^{n}$ has domain a finite subset of $\kappa$, there is an $\alpha<\kappa$ such that in fact

$\mathbf{1}_{\mathbb{P}(\alpha)} \|-$ "Each $\dot{\mathcal{U}}_{n}$ is a countable cover by ground-model open sets".

Let $G$ be $\mathbb{P}(\kappa)$-generic. Then $G_{\alpha}=\left\{p\left\lceil_{\alpha}: p \in G\right\}\right.$ is $\mathbb{P}(\alpha)$-generic over $V$. In $V\left[G_{\alpha}\right]$ write $\mathcal{U}_{n}=\left(U_{m}^{n}: m \in \mathbb{N}\right)$. Define, for each $x \in X$, the function 
$f_{x} \in \mathbb{N}_{\mathbb{N}}$ by $f_{x}(n)=\min \left\{m: x \in U_{m}^{n}\right\}$. Now $\left\{f_{x}: x \in X\right\} \in V\left[G_{\alpha}\right]$. If $c \in V[G]$ is any Cohen real over $V\left[G_{\alpha}\right]$, then for each $x$ in $X,\{n \in \mathbb{N}$ : $\left.c(n)=f_{x}(n)\right\}$ is infinite, and thus $\left(U_{c(n)}^{n}: n \in \mathbb{N}\right)$ witnesses $\mathbf{S}_{1}^{\omega}(\mathcal{O}, \mathcal{O})$ for $X$ in $V[G]$.

Thus, forcing with a sufficient number of Cohen reals preserves being a Rothberger space, but fails to preserve not being Rothberger.

Problem 2. Is it consistent that adjoining a single Cohen real converts each ground-model Lindelöf space to a Rothberger space or at least makes it indestructible?

COROllary 12 (A. Dow). Adjoining $\aleph_{1}$ Cohen reals renders each groundmodel Lindelöf space indestructible.

Proof. By Theorem 11 each ground-model Lindelöf space acquires the Rothberger property. By Theorem 9 in the extension ONE has no winning strategy in the game $\mathrm{G}_{1}^{\omega_{1}}(\mathcal{O}, \mathcal{O})$. Apply Theorem 1 .

Dow's proof in [13] uses a quite nontrivial elementary submodel argument.

Rothberger spaces and random reals. Let $\mathbb{B}(\kappa)$ be the partially ordered set for adding $\kappa$ random reals. We now show that forcing with $\mathbb{B}(\kappa)$ preserves being Rothberger. In contrast to the case of Cohen reals, for a large class of Lindelöf spaces random reals preserve not being Rothberger. In the proof that adding $\kappa$ random reals preserves the Rothberger property we make use of the fact that the Lindelöf property is preserved, as was noted in the remarks in and following the proof of Corollary 2.5 of [20]. Since the proof of the latter fact does not appear explicitly in the literature, the referee requested that we include a proof here.

THEOREM 13. If $X$ is a Lindelöf space, then

$$
\mathbf{1}_{\mathbb{B}(\kappa)} \|- \text { " } \check{X} \text { is a Lindelöf space". }
$$

Proof. Given an open cover $\mathcal{U}$ in the extension, we may assume without loss of generality that it is composed of ground-model open sets. For each $x \in X$ pick a maximal antichain $A_{x}$ in $\mathbb{B}(\kappa)$ and ground-model open sets $U_{p, x}$ containing $x$, for each $p \in A_{x}$, such that $p \|-$ " $\check{U}_{p, x} \in \dot{\mathcal{U}}$ ". For each positive integer $n$ choose a finite set $A_{n, x} \subseteq A_{x}$ such that $\mu\left(\bigcup A_{n, x}\right)>1-1 / n$ and define

$$
V_{n, x}=\bigcap\left\{U_{p, x}: p \in A_{n, x}\right\} .
$$

Then $\left\{V_{n, x}: x \in X\right\}$ is an open cover of $X$ in the ground model, and thus has a countable subset $\mathcal{V}_{n}$ that covers $X$.

Claim. $\mathbf{1}_{\mathbb{B}(\kappa)} \|-"(\forall y \in \check{X})(\exists n<\check{\omega})\left(\exists V \in \check{\mathcal{V}}_{n}\right)(\exists U \in \dot{\mathcal{U}})(y \in V \subseteq U)$ ". 
Proof of Claim. We have to prove that $(\forall y \in X)(\forall q)(\exists r \leq q)(\exists n \in \omega)\left(\exists V \in \mathcal{V}_{n}\right)(\exists U$ open $)(r \|-$ " $\check{y} \in \check{V} \subseteq \check{U} \in \dot{\mathcal{U}} ")$. Fix $y \in X$ and $q \in \mathbb{B}(\kappa)$. Take $n$ so large that $\mu(q)>1 / n$. Since $\mathcal{V}_{n}$ covers $X$, choose $V_{n, x} \in \mathcal{V}_{n}$ with $y \in V_{n, x}$. By construction there is a $p \in A_{n, x}$ with $p$ and $q$ compatible. Choose $r \leq p, q$. Then $r \|-y \in \check{V}_{n, x} \subseteq \check{U}_{p, x} \in \dot{\mathcal{U}}$ ", completing the proof of the Claim.

Let $G$ be $\mathbb{B}(\kappa)$-generic over $V$. By the Claim in $V[G]$, for each $y \in X$ choose $V_{n, x}^{y}$ such that $y \in V_{n, x}^{y} \subseteq U$ for some $U \in \mathcal{U}$. Then $\left\{V_{n, x}^{y}: y \in Y\right\}$ $\subseteq \bigcup_{m<\omega} \mathcal{V}_{m}$ is a countable refinement of $\mathcal{U}$ and covers $X$. Thus $\mathcal{U}$ has a countable subset that covers $X$.

Lemma 14. Let $X$ be a Lindelöf space and let $\dot{\mathcal{U}}$ be a $\mathbb{B}(\kappa)$-name such that

$$
\mathbf{1}_{\mathbb{B}(\kappa)} \|-" \dot{\mathcal{U}} \text { is an open cover of } \check{X} " .
$$

Then for each $x \in X$ and each $k<\infty$ there is a neighborhood $N_{k}(x)$ of $x$ such that

$$
\mu\left(\left\|(\exists U \in \dot{\mathcal{U}})\left(\check{N}_{k}(x) \subseteq U\right)\right\|\right)>1-\frac{1}{2^{k+1}} .
$$

Proof. Since $X$ is Lindelöf and random reals preserve Lindelöf, and since the ground-model open sets form a basis of the topology $X$ has in the generic extension, we may assume that

$\mathbf{1}_{\mathbb{B}(\kappa)} \|-" \dot{\mathcal{U}}$ is a countable open cover of $\check{X}$ by ground-model sets" and choose $\mathbb{B}(\kappa)$-names $\dot{U}_{n}, n<\infty$, such that

$\mathbf{1}_{\mathbb{B}(\kappa)} \|-" \dot{\mathcal{U}}=\left\{\dot{U}_{n}: n<\omega\right\}$ and each $\dot{U}_{n}$ is in the ground model".

We may assume each $\dot{U}_{n}$ is of the form $\left\{\left(\check{U}_{k}^{n}, b_{k}^{n}\right): k<\omega\right\}$ where for each $n$ and each $k, U_{k}^{n}$ is a ground-model open set and $\left\{b_{k}^{n}: k<\infty\right\}$ is a maximal antichain of $\mathbb{B}(\kappa)$.

Now consider $x \in X$. For each $n$ and $k$ define

$$
V_{k}^{n}(x)= \begin{cases}X & \text { if } x \notin U_{k}^{n}, \\ U_{k}^{n} & \text { otherwise. }\end{cases}
$$

For each $k$ define $M_{k}(x)=\bigcap_{i, j \leq k} V_{j}^{i}(x)$. Note that

$$
c_{k}=\left\|(\exists i \leq k)\left(\check{M}_{k}(x) \subseteq \dot{U}_{i}\right)\right\| \geq \sup \left\{b_{j}^{i}: i, j \leq k \text { and } x \in U_{j}^{i}\right\} .
$$

For $k<\ell$ we have $\mu\left(c_{k}\right) \leq \mu\left(c_{\ell}\right)$, and since $\mu(\| \dot{\mathcal{U}}$ is a cover $\|)=1$ we have $\lim _{k \rightarrow \infty} \mu\left(c_{k}\right)=1$. For each $m$ choose $k_{m}$ so large that $\mu\left(c_{k_{m}}\right)>1-1 / 2^{m+1}$ and put $N_{m}(x)=M_{k_{m}}(x)$.

TheOREM 15. If $X$ is a Rothberger space then $\mathbf{1}_{\mathbb{B}(\kappa)} \|-$ " $\check{X}$ is a Rothberger space". 
Proof. Choose $\mathbb{B}(\kappa)$-names $\dot{\mathcal{U}}_{n}$ such that $\mathbf{1}_{\mathbb{B}(\kappa)} \|-"\left(\dot{\mathcal{U}}_{n}: n<\infty\right)$ is a sequence of open covers of $\check{X}$ ".

By Lemma 14, for each $n$ and each $x \in X$ choose a neighborhood $W_{n}(x)$ such that $\mu\left(\left\|\left(\exists U \in \dot{\mathcal{U}}_{n}\right)\left(\check{W}_{n}(x) \subseteq U\right)\right\|\right)>1-1 / 2^{n+1}$. Then $\mathcal{W}_{n}=\left\{W_{n}(x)\right.$ : $x \in X\}$ is an open cover of $X$. In the ground model apply the fact that $X$ is Rothberger to the sequence $\left(\mathcal{W}_{n}: n<\infty\right)$ of open covers of $X$. For each $n$ choose a set $S_{n} \in \mathcal{W}_{n}$ such that for each $x \in X$ there are infinitely many $n$ with $x \in S_{n}$.

Observe that $\mu\left(\left\|\left(\exists U \in \dot{\mathcal{U}}_{n}\right)\left(\check{S}_{n} \subseteq U\right)\right\|\right)>1-1 / 2^{n+1}$ for each $n$. Thus, choose by the Fullness Lemma (see [24, Lemma 18.6]) for each $n$ a $\mathbb{B}(\kappa)$-name $\dot{U}_{n}$ such that $\mu\left(\| \dot{U}_{n} \in \dot{\mathcal{U}}_{n}\right.$ and $\left.\check{S}_{n} \subseteq \dot{U}_{n} \|\right) \geq 1-1 / 2^{n+1}$.

Claim. $\mathbf{1}_{\mathbb{B}(\kappa)} \|-" \check{X} \subseteq \bigcup_{n<\infty} \dot{U}_{n} "$.

For suppose not and choose $b$ so that $b \|-$ " $\check{X} \nsubseteq \bigcup_{n<\infty} \dot{U}_{n}$ ". Choose $c<b$ and $x \in X$ so that $c \|-" \check{x} \notin \bigcup_{n<\infty} \dot{U}_{n} "$. Then choose $n$ so large that $\mu(c)>1 / 2^{n}$. Choose a $k$ larger than $n$ with $x \in S_{k}$. Now define

$$
d=c \wedge \| \text { " } \dot{U}_{k} \in \dot{\mathcal{U}}_{k} \text { and } \check{S}_{k} \subseteq \dot{U}_{k} " \| .
$$

We have $\mu(d)>0$ and since $d \leq c$ we have $d \|-" \check{x} \notin \bigcup_{n<\infty} \dot{U}_{n}$ ". But also $x \in S_{k}$ and $d \|-" \check{S}_{k} \subseteq \dot{U}_{k}$ ", a contradiction. The proof is complete.

Next we examine preservation of non-Rothberger. Let $n$ be a positive integer. A family $\mathcal{A}$ of subsets of a set $X$ has degree $n$ if $n$ is the smallest positive integer such that for each $x \in X$ the set $\{A \in \mathcal{A}: x \in A\}$ has cardinality at most $n$. We shall say that a topological space is $n$-dimensional if $n$ is the smallest nonnegative integer such that each open cover of the space has an open refinement of degree $n+1$. And a space is said to be strongly countable-dimensional if it is a union of countably many closed subsets, each of finite dimension. This notion was introduced by Nagata [36] and Smirnov [48, independently.

If a strongly countable-dimensional space does not have the Rothberger property, then some closed subset of finite dimension does not have the Rothberger property. This follows from the fact that the Rothberger property is inherited by closed subspaces, and since a union of countably many subspaces, each with the Rothberger property, again has the Rothberger property. We now show that for strongly countable-dimensional ground-model spaces random reals preserve the property of not being a Rothberger space. In the process we use the following fact (see "equation" (1) in the proof of Theorem 17 below): If a space $X$ has the Rothberger property and if a sequence $\left(\mathcal{U}_{n}: n<\infty\right)$ of open covers is given, then there is a sequence $\left(U_{n}: n<\infty\right)$ such that for each $n$ we have $U_{n} \in \mathcal{U}_{n}$ and for each $x \in X$ there are infinitely many $n$ with $x \in U_{n}$ : first write the natural numbers as 
a union of countably many disjoint infinite sets $Y_{k}, k<\infty$, and apply the Rothberger property to each subsequence $\left(\mathcal{O}_{n}: n \in Y_{k}\right)$.

Lemma 16. If $\dot{U}$ is a $\mathbb{B}(\kappa)$-name and $A$ and $B$ are ground-model sets such that

$$
\mu(\|\dot{U}=\check{A}\| \wedge\|\dot{U}=\check{B}\|)>0
$$

then $A=B$.

Proof. Consider a generic filter $G$ which contains $\|\dot{U}=\check{A}\| \wedge\|\dot{U}=\check{B}\|$. In the generic extension, $A=\check{A}_{G}=\dot{U}_{G}=\check{B}_{G}=B$. But $A$ and $B$ are ground-model sets.

THEOREM 17. Let $\kappa$ be a cardinal number. If $X$ is a strongly countable dimensional Lindelöf space and if

$$
\mathbf{1}_{\mathbb{B}(\kappa)} \|- \text { "X has the Rothberger property", }
$$

then $X$ has the Rothberger property in the ground model.

Proof. Suppose that $\mathbf{1}_{\mathbb{B}(\kappa)} \|-$ " $\check{X}$ has the Rothberger property" while, in fact, $X$ does not have the Rothberger property. Choose a positive integer $m$ and a closed subset $Y$ of $X$ which is $(m-1)$-dimensional, but does not have the Rothberger property. Let $\left(\mathcal{O}_{n}: n<\infty\right)$ be a sequence of open covers of $Y$ witnessing this. We may assume each $\mathcal{O}_{n}$ has degree $m$, and that each $\mathcal{O}_{n+1}$ refines $\mathcal{O}_{n}$. Enumerate each $\mathcal{O}_{n}$ bijectively as $\left(U_{k}^{n}: k \in I_{n}\right)$, where $I_{n}$ is either an initial segment of the set of natural numbers, or the set of natural numbers. Note that

$$
\mathbf{1}_{\mathbb{B}(\kappa)} \|-" \check{Y} \text { is closed in } \check{X} \text {, thus has the Rothberger property". }
$$

For each $n$, put $\epsilon_{n}=1 /\left((n+1)^{2} \dot{m}\right)$. Define $\psi: \mathbb{N} \rightarrow \mathbb{N}$ as follows: $\psi(1)=1 / \epsilon_{1}$ and for each $n, \psi(n+1)=\psi(n)+1 / \epsilon_{n+1}$.

In $V^{\mathbb{B}(\kappa)}$ look at $\left(\check{\mathcal{O}}_{\psi(n)}: n<\infty\right)$, a sequence of open covers of $Y$. Since $\mathbf{1}_{\mathbb{B}(\kappa)} \|-$ " $\check{Y}$ has the Rothberger property", choose a sequence $\left(\dot{V}_{n}: n<\infty\right)$ of $\mathbb{B}(\kappa)$-names such that

$$
\mathbf{1}_{\mathbb{B}(\kappa)} \|-"(\forall n)\left(\emptyset \neq \dot{V}_{n} \in \check{\mathcal{O}}_{\psi(n)}\right) \text { and }(\forall x \in \check{Y})\left(\exists_{n}^{\infty}\right)\left(x \in \dot{V}_{n}\right) " .
$$

For each $n$ define

$$
\mathcal{W}_{n}:=\left\{O_{j}^{\psi(n)} \in \mathcal{O}_{\psi(n)}: \mu\left(\left\|\dot{V}_{n}=\check{O}_{j}^{\psi(n)}\right\|\right)>\epsilon_{n}\right\} .
$$

Since elements of $\mathcal{O}_{\psi(n)}$ are listed without repetition, Lemma 16 implies that $C_{n}=\left|\mathcal{W}_{n}\right| \leq m \cdot(n+1)^{2}$. List the elements of each $\mathcal{W}_{n}$ as $\left(O_{m_{j}^{n}}^{\psi(n)}, 1 \leq j\right.$ $\left.\leq C_{n}\right)$.

Using the fact that for each $n, \mathcal{O}_{n+1}$ refines $\mathcal{O}_{n}$, we can choose $O_{m_{n}}^{n} \in \mathcal{O}_{n}$, $n<\infty$, such that there is for each $U \in \bigcup_{n<\infty} \mathcal{W}_{n}$ an $n$ with $U \subseteq O_{m_{n}}^{n}$. 
Since $\left(\mathcal{O}_{n}: n<\infty\right)$ witnessed that $Y$ does not have the Rothberger property, choose

$$
x \in Y \backslash \bigcup_{n<\infty} O_{m_{n}}^{n} .
$$

Claim 1. For each $n, \mu\left(\left\|\check{x} \in \dot{V}_{n}\right\|\right) \leq 1 /(n+1)^{2}$.

Proof of the Claim. If not, choose $n$ with $\mu\left(\left\|\check{x} \in \dot{V}_{n}\right\|\right)>1 /(n+1)^{2}$. Since $\mathcal{O}_{\psi(n)}$ covers $Y$, the set $I=\left\{j: x \in O_{j}^{\psi(n)}\right\}$ is nonempty and for each $j \in I$ we have $\mu\left(\left\|\check{x} \in \check{O}_{j}^{\psi(n)}\right\|\right)=1$, and so $\mu\left(\left\|\check{x} \in \dot{V}_{n} \cap \bigcap_{j \in I} \check{O}_{j}^{\psi(n)}\right\|\right)>$ $1 /(n+1)^{2}$. But then as $\mu\left(\left\|\dot{V}_{n} \in \check{\mathcal{O}}_{\psi(n)}\right\|\right)=1$ we have

$$
\frac{1}{(n+1)^{2}}<\mu\left(\left\|\dot{V}_{n} \in\left\{\check{O}_{j}^{\psi(n)}: j \in I\right\}\right\|\right)
$$

and $\mathcal{O}_{\psi(n)}$ has degree $m$, for some $j \in I$ we have $\mu\left(\left\|\dot{V}_{n}=\check{O}_{j}^{\psi(n)}\right\|\right)>$ $1 /\left(m \cdot(n+1)^{2}\right)$ and so for this $j, O_{j}^{\psi(n)} \in \mathcal{W}_{n}$, contradicting the choice of $x$. Claim 1 is proven.

Consider any $b \in \mathbb{B}(\kappa)$ with $\mu(b)>0$, and choose $n$ so large that $\mu(b)>$ $\sum_{j=n}^{\infty} 1 / j^{2}$. With Claim 1 proven, define

$$
a=b \backslash \bigvee_{k \geq n}\left\|\check{x} \in \dot{V}_{k}\right\| .
$$

We have $a<b, \mu(a)>0$ and

$$
a \|-"(\forall k \geq n)\left(\check{x} \notin \dot{V}_{k}\right) " .
$$

But then (2) contradicts (1). Thus, $X$ also has the Rothberger property.

In our first proof of Theorem 17 we used the hypothesis that the space is zero-dimensional. Subsequently we improved the hypothesis that the space be zero-dimensional to the current hypothesis of strong countable dimensionality. We were not able to eliminate the dimension-theoretic hypothesis altogether from Theorem 17 .

Problem 3. Is the dimension hypothesis in Theorem 17 necessary?

A large class of topological spaces is covered by the zero-dimensional case of Theorem 17 ,

LEMma 18. $\mathrm{T}_{3}$ spaces with the property $\mathrm{S}_{1}^{\omega}(\mathcal{O}, \mathcal{O})$ are zero-dimensional.

Proof. If $X$ is $\mathrm{T}_{3}$ and Lindelöf, it is $\mathrm{T}_{4}$ and thus $\mathrm{T}_{3 \frac{1}{2}}$. Since $X$ is $\mathrm{T}_{3 \frac{1}{2}}$ fix an embedding of $X$ into a power of the unit interval $I$, say $F: X \rightarrow I^{\kappa}$. For each $\alpha \in \kappa$ the projection $\pi_{\alpha}$ onto the $\alpha$ th coordinate is continuous, and so $\pi_{\alpha} \circ F[X] \subseteq[0,1]$ has property $\mathrm{S}_{1}^{\omega}(\mathcal{O}, \mathcal{O})$, and thus is zero-dimensional. But then $\prod_{\alpha<\kappa} \pi_{\alpha} \circ F[X]$ is zero-dimensional. Since the latter set contains $F[X]$, it follows that $F[X]$, and thus $X$, is zero-dimensional. 
It is easy to convert, by forcing, certain ground-model spaces to spaces which have dimension zero in the generic extension. In the generic extension by one Cohen real every ground-model separable metric space acquires dimension zero. To see this, first observe that the real line of the ground model acquires Lebesgue measure zero: Temporarily let $\mu$ denote Lebesgue measure and let $\mu^{*}$ denote the outer measure. In the ground model choose for each $\epsilon>0$ a sequence $\left(\epsilon_{n}: n<\omega\right)$ of positive reals such that $\sum_{n<\omega} \epsilon_{n}<\epsilon$. We show that

$$
\mathbf{1}_{\mathbb{P}(\omega)} \|-“ \mu(\check{\mathbb{R}})=0 " .
$$

If not, choose $p \in \mathbb{P}(\omega)$ and a positive integer $n$ such that $p \|-" \mu^{*}(\check{\mathbb{R}})>$ $1 / \check{n} "$. Consider in the ground model the sequence $\left(\epsilon_{m}: m<\omega\right)$ associated with $1 / n=\epsilon$, and for each $m$ define $\mathcal{U}_{m}=\left\{J_{k}^{m}: k<\omega\right\}$ where $\left\{J_{k}^{m}\right.$ : $k<\omega\}$ enumerates the set of intervals of length less than $\epsilon_{m}$ which have rational endpoints. For each $x \in \mathbb{R}$ define $f_{x}$ so that for all $m, f_{x}(m)=$ $\min \left\{k: x \in J_{k}^{m}\right\}$. Note that each $f_{x}$ is in the ground model. For each $x \in \mathbb{R}$ and $m>|p|$ the set $D_{m}^{x}=\left\{q \in \mathbb{P}(\omega):(\exists k>m)\left(f_{x}(k)=q(k)\right\}\right.$ is dense in $\mathbb{P}(\omega)$, and thus dense below $p$. Then any generic filter containing $p$ meets each $D_{m}^{x}$, and so the Cohen real obtained from this generic filter, say its $\mathbb{P}(\omega)$-name is $\dot{f}$, is infinitely often equal to each $f_{x}$. This implies

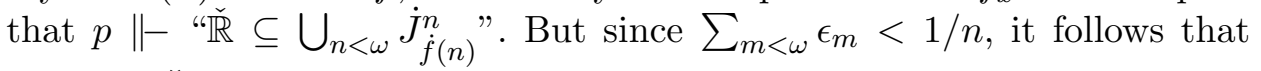
$p \|-" \mu^{*}(\check{\mathbb{R}})<1 / \check{n} "$, a contradiction.

Since Lebesgue measure zero sets of real numbers are zero-dimensional, it follows that the ground-model set of reals is zero-dimensional in the generic extension. But then the Hilbert cube of the ground model is in the extension a product of zero-dimensional spaces and so is zero-dimensional. Separable metric spaces from the ground model embed homeomorphically into the Hilbert cube of the ground model, thus in the generic extension are subspaces of a zero-dimensional space and so themselves are zero-dimensional.

This specific consequence of Cohen real forcing holds more generally.

Corollary 19. If $X$ is a $\mathrm{T}_{3}$ Lindelöf space and $\kappa$ is any cardinal number, then $\mathbf{1}_{\mathbb{P}(\kappa)} \|-$ "X is zero-dimensional".

Similar ideas give the following result for spaces not necessarily Lindelöf $(2)$.

Corollary 20. Let $M$ and $N$ be models with $M \subseteq N$ and with $X$ a $\mathrm{T}_{3 \frac{1}{2}}$ space in $M$. Assume there is a real number in $N$ not in $M$. Then $N \models$ " $X$ is zero-dimensional".

Proof. The ground-model reals are zero-dimensional in $N$ since between any two rationals, there is a new real. Within $M$ embed $X$ into a power

$\left({ }^{2}\right)$ We thank the referee for this improvement of our earlier version of Corollary 20. 
of $[0,1]$. In $N$ this is a power of a zero-dimensional space, so zero-dimensional. But then in $N, X$ is homeomorphic to a zero-dimensional space.

Rothberger spaces and countably closed forcing. Since Rothberger spaces are indestructibly Lindelöf, they remain Lindelöf under countably closed forcing. The following result shows a little more:

TheOREM 21. Let $(\mathbb{P},<)$ be a countably closed partially ordered set. For $X$ a space the following are equivalent:

1. $X$ is a Rothberger space.

2. $\mathbf{1}_{\mathbb{P}} \|-$ " $\check{X}$ is a Rothberger space".

Proof. $1 \Rightarrow 2$ : Let $(X, \mathcal{T})$ be a space which has property $\mathrm{S}_{1}^{\omega}(\mathcal{O}, \mathcal{O})$. Let $\left(\dot{\mathcal{U}}_{n}: n \in \mathbb{N}\right)$ be a name for a sequence of open covers of $X$. That is,

$1_{\mathbb{P}} \|-$ " $\left(\dot{\mathcal{U}}_{n}: n \in \mathbb{N}\right)$ is a sequence of open covers for $\check{X} "$.

Let $G$ be $\mathbb{P}$-generic and in $V[G]$ consider the sequence of open covers. Since $X$ was, in the ground model, $\mathrm{S}_{1}^{\omega}(\mathcal{O}, \mathcal{O})$, it is in $V[G]$ still Lindelöf. We may also assume that each $\mathcal{U}_{n}$ consists of sets from the ground-model topology $\mathcal{T}$ on $X$. Thus, for each $n$ there is in $V[G]$ a function $f_{n}: \omega \rightarrow \mathcal{T}$ such that $\mathcal{U}_{n}=f_{n}[\omega]$. Since the forcing is countably closed, each $f_{n}$ is a member of the ground model, and thus each $\mathcal{U}_{n}$ is a member of the ground model. But then apply $\mathrm{S}_{1}^{\omega}(\mathcal{O}, \mathcal{O})$ in the ground model to this sequence to obtain $U_{n} \in \mathcal{U}_{n}$, $n<\infty$, such that $\left\{U_{n}: n<\infty\right\}$ is a cover of $X$.

$2 \Rightarrow 1$ : Assume that $\mathbf{1}_{\mathbb{P}} \|-$ " $\check{X}$ is a Rothberger space". Suppose that contrary to item 1 , in the ground model $X$ is not a Rothberger space. Choose in the ground model a sequence $\left(\mathcal{U}_{n}: n<\infty\right)$ of open covers of $X$ witnessing that $X$ is not Rothberger. By item 2 , choose a $\mathbb{P}$-name $\dot{f}$ such that $\mathbf{1}_{\mathbb{P}} \|-" \dot{f}: \check{\omega} \rightarrow \bigcup_{n<\infty} \check{\mathcal{U}}_{n}$ and $(\forall n)\left(\dot{f}(n) \in \check{\mathcal{U}}_{n}\right)$ and $\{\dot{f}(n): n \in \check{\omega}\}$ covers $\check{X} "$. Choose for each $n$ a $p_{n} \in \mathbb{P}$ and a $U_{n} \in \mathcal{U}_{n}$ such that $p_{n+1}<p_{n}$ and $p_{n} \|-" \dot{f}(\check{n})=\check{U}_{n} "$. Since $\mathbb{P}$ is countably closed choose $p \in \mathbb{P}$ such that for all $n$ we have $p<p_{n}$. Then $p \|-"(\forall n)\left(\check{U}_{n} \in \check{\mathcal{U}}_{n}\right.$ and $\left.\check{X}=\bigcup_{n<\infty} \check{U}_{n}\right) "$. But all parameters in the statement forced by $p$ are in the ground model; thus the statement is true in the ground model. This contradicts that the sequence $\left(\mathcal{U}_{n}: n<\infty\right)$ of open covers of $X$ witnesses that $X$ is not Rothberger.

Cardinality upper bounds on points $\mathrm{G}_{\delta}$ Rothberger spaces. It is consistent that all metrizable Rothberger spaces are countable. This follows from the Borel Conjecture. Carlson [10] showed that the Borel Conjecture is equivalent to the statement that all strong measure zero metric spaces are countable. Thus the Borel Conjecture implies that all metrizable Rothberger spaces are countable. Sierpiński [47] proved that the Continuum Hypothesis implies the negation of the Borel Conjecture. R. Laver [32] proved that the 
Borel Conjecture is consistent relative to the consistency of ZFC only. In Laver's model, $2^{\aleph_{0}}=\aleph_{2}$.

Proposition 22 (Judah, Shelah, Woodin). For each cardinal number $\kappa$ it is consistent that $2^{\aleph_{0}}>\kappa$ and every metrizable Rothberger space is countable.

Proof. Consider a model of Borel's Conjecture. By Theorem 17 we know that upon forcing with $\mathbb{B}(\kappa)$ every ground-model set of real numbers with the Rothberger property is countable. It is not known if uncountable metric Rothberger spaces can be introduced by random real forcing. But by a result of Judah, Shelah and Woodin [25] if the ground model is Laver's model, then subsequent forcing with $\mathbb{B}(\kappa)$ introduces no uncountable metrizable Rothberger spaces.

COROLlary 23. If it is consistent that there is a real-valued measurable cardinal then it is consistent that each metrizable Rothberger space is countable and there is a real-valued measurable cardinal $\leq 2^{\aleph_{0}}$.

Proof. Assume it is consistent that there is a measurable cardinal, $\kappa$. Then it is consistent that $\kappa$ is measurable and $\mathrm{CH}$ holds (collapse the continuum to $\aleph_{1}$ with countable conditions, if necessary, and apply [33, Theorem 3]: "Mild" forcing preserves measurability.). Then use Laver's method to force Borel's Conjecture. By [33, Theorem 3], $\kappa$ still is measurable. Now force with $\mathbb{B}(\kappa)$. As noted in the proof of Proposition 22, in the generic extension all metrizable Rothberger spaces are countable. By a theorem of Solovay $\kappa=2^{\aleph_{0}}$ is real-valued measurable.

Arhangel'skii's result trivially implies that if $\kappa$ is the least measurable cardinal, then each points $\mathrm{G}_{\delta}$ Rothberger space is of cardinality $<\kappa$. We shall see in Proposition 27 that this upper bound cannot be lowered in ZFC. But a better theorem can be proved for the points $G_{\delta}$ Rothberger spaces:

THEOREM 24. If a space has the Rothberger property and each point is a $\mathrm{G}_{\delta}$ then its cardinality is less than the smallest real-valued measurable cardinal.

Proof. Suppose that $\kappa$ is a real-valued measurable cardinal and that $X$ is a Lindelöf space with all points $\mathrm{G}_{\delta}$ and $|X| \geq \kappa$. Choose a subset $Y$ of $X$ with $|Y|=\kappa$, and let $\mu: \mathcal{P}(Y) \rightarrow[0,1]$ be a countably additive atomless measure with $\mu(Y)=1$. For each $x \in X$ choose a sequence $\left(U_{n}(x): n<\infty\right)$ of neighborhoods with $\{x\}=\bigcap_{n<\infty} U_{n}(x)$ and $U_{n+1}(x) \subseteq U_{n}(x)$ for all $n$.

For each $m$, choose for each $x$ an $n(m, x)$ such that $\mu\left(Y \cap U_{n(m, x)}(x)\right)<$ $1 / 2^{m+2}$. Then

$$
\mathcal{U}_{m}=\left\{U_{n(m, x)}(x): x \in X\right\}
$$

is an open cover of $X$. For the sequence $\left(\mathcal{U}_{m}: m<\infty\right)$ of open covers of $X$, 
consider any sequence $\left(U_{m}: m<\infty\right)$ with $U_{m} \in \mathcal{U}_{m}$ for each $m$. Then $\mu\left(Y \cap U_{m}\right)<1 / 2^{m+2}$ for each $m$, and so

$$
\mu\left(Y \cap \bigcup_{m<\infty} U_{m}\right) \leq \sum_{m=1}^{\infty} \frac{1}{2^{m+2}}<\frac{1}{2}<\mu(Y) .
$$

But then the sequence $\left(U_{m}: m<\infty\right)$ does not cover $Y$ and so not $X$. It follows that $X$ is not Rothberger.

The bound given in Theorem 24 cannot be lowered in ZFC:

THEOREM 25. If it is consistent that there is a real-valued measurable cardinal, then it is consistent that there is a real-valued measurable cardinal $\kappa \leq 2^{\aleph_{0}}$ and for each cardinal $\lambda<\kappa$ there is a space $X$ with points $\mathrm{G}_{\delta}$ and the Rothberger property, with $\lambda \leq|X|<\kappa$.

Proof. If it is consistent that there is a real-valued measurable cardinal, then it is consistent that there is a measurable cardinal [49]. Thus, assume that $\kappa$ is the least measurable cardinal. There are Lindelöf spaces with points $\mathrm{G}_{\delta}$ of arbitrarily large cardinality below $\kappa$, for example Juhász's spaces described in Section 4, Example 4. First force with $\aleph_{1}$ Cohen reals. Then by Theorem 11 the ground-model versions of Juhász's spaces are Rothberger spaces with points $\mathrm{G}_{\delta}$ of arbitrarily large cardinality below $\kappa$. By Theorem 3 of [33], $\kappa$ is still measurable in this generic extension. Now force with $\mathbb{B}(\kappa)$ and apply Solovay's theorem [49] that $\mathbf{1}_{\mathbb{B}(\kappa)} \|-{ }^{2} 2^{\aleph_{0}}=\check{\kappa}$ is real-valued measurable".

In the resulting model $2^{\aleph_{0}}$ is real-valued measurable and there are Rothberger spaces with points $\mathrm{G}_{\delta}$ of arbitrarily large cardinality below $2^{\aleph_{0}}$.

Note that in the proof of Theorem 25 we may also force with $\mathbb{B}(\nu)$ for $\nu>\kappa$, thus causing the least real-valued measurable cardinal to be less than $2^{\aleph_{0}}$.

Theorem 24also provides an alternative proof to Arhangel'skii's theorem:

COROLlary 26 (Arhangel'skii). Every points $\mathrm{G}_{\delta}$ Lindelöf space has cardinality less than the least (two-valued) measurable cardinal.

Proof. Assume that on the contrary $\kappa$ is the least two-valued measurable cardinal and that $X$ is a points $\mathrm{G}_{\delta}$ space of cardinality at least $\kappa$. First force with $\aleph_{1}$ Cohen reals, converting $X$ to a Rothberger space while preserving the measurability of $\kappa$. Now force with $\kappa$ random reals, converting $\kappa$ into a real-valued measurable cardinal while preserving the Rothberger property of $X$. This gives a contradiction to the result of Theorem 24 .

Let us also note that in the absence of real-valued measurable cardinals, the least measurable cardinal may be the least upper bound on the cardinality of points $\mathrm{G}_{\delta}$ Rothberger spaces: 
Proposition 27. If it is consistent that there is a measurable cardinal, then it is consistent that there is a measurable cardinal $\kappa$ and for each $\lambda<\kappa$ there is a points $\mathrm{G}_{\delta}$ Rothberger space of cardinality at least $\lambda$.

Proof. Assume that $\kappa$ is the least measurable cardinal. There are Lindelöf spaces with points $\mathrm{G}_{\delta}$ of arbitrarily large cardinality below $\kappa$, for example Juhász's spaces described in Section 4, Example 4. First force with $\aleph_{1}$ Cohen reals. Then by Theorem 11 the ground-model versions of Juhász's spaces are Rothberger spaces with points $\mathrm{G}_{\delta}$ of arbitrarily large cardinality below $\kappa$, and by Theorem 3 of [33], $\kappa$ is still measurable in this generic extension.

It is a trivial consequence of Tall's result, Theorem 7 , that if it is consistent that there is a supercompact cardinal then it is consistent that all points $G_{\delta}$ Rothberger spaces have cardinality $\leq \aleph_{1}$ and $2^{\aleph_{0}}=\aleph_{1}$. However, we do not know if either of the following is possible (even assuming the consistency of appropriate large cardinals):

Problem 4. Is it possible that all points $\mathrm{G}_{\delta}$ Rothberger spaces have cardinality $\leq \aleph_{1}$ while there is an indestructible Lindelöf space with points $\mathrm{G}_{\delta}$ and of cardinality larger than $\aleph_{1}$ ?

Proposition 28 records that if it is consistent that there is a measurable cardinal, then it is consistent that every points $G_{\delta}$ Rothberger space has cardinality less than $2^{\aleph_{0}}$ and there are points $\mathrm{G}_{\delta}$ Lindelöf spaces of arbitrarily large cardinality below the first (two-valued) measurable cardinal.

Proposition 28. Assume that it is consistent that there is a measurable cardinal. Then the conjunction of the following list of statements is consistent:

1. Every Rothberger space with points $\mathrm{G}_{\delta}$ is of cardinality less than $2^{\aleph_{0}}$.

2. For each cardinal $\lambda<2^{\aleph_{0}}$ there is a Rothberger space with points $\mathrm{G}_{\delta}$ such that $\lambda<|X|<2^{\aleph_{0}}$.

3. For each cardinal $\lambda>2^{\aleph_{0}}$ which is less than the first measurable cardinal there is a points $\mathrm{G}_{\delta}$ Lindelöf space $X$ such that $\lambda<|X|$.

Problem 5. Is it possible that all points $\mathrm{G}_{\delta}$ Rothberger spaces have cardinality $\leq \aleph_{1}$ while $2^{\aleph_{0}}>\aleph_{1}$ ?

Since the real line is indestructibly Lindelöf, a positive answer to Problem 5 gives a positive answer to Problem 4 .

When Lindelöf implies Rothberger. In Theorem 47 we shall see a topological hypothesis under which Lindelöf implies Rothberger (and more). Here we explore set-theoretic hypotheses that imply the equivalence of the Lindelöf and Rothberger properties. Let $\mathcal{M}_{\mathbb{R}}$ denote the collection of first 
category subsets of the real line. Then $\operatorname{cov}\left(\mathcal{M}_{\mathbb{R}}\right)$ denotes the least cardinality of a family of first category sets whose union covers the real line.

Proposition 29. For an infinite cardinal number $\kappa$ the following are equivalent:

1. Every Lindelöf space of cardinality at most $\kappa$ has the Rothberger property.

2. $\kappa<\operatorname{cov}\left(\mathcal{M}_{\mathbb{R}}\right)$.

Proof. $2 \Rightarrow 1$ : Let $X$ be a Lindelöf space of cardinality $\kappa$. Let $\left(\mathcal{U}_{n}: n<\infty\right)$ be a sequence of open covers of $X$. Since $X$ is Lindelöf we may assume each $\mathcal{U}_{n}$ is countable and enumerate it as $\left(U_{k}^{n}: k<\infty\right)$. For each $x$ in $X$ define

$$
N_{x}=\left\{f \in{ }^{\omega} \omega:(\forall n)\left(x \notin U_{f(n)}^{n}\right)\right\} .
$$

Each $N_{x}$ is nowhere dense in ${ }^{\omega} \omega$ with the usual product topology, which is homeomorphic to the set of irrational numbers. Then $\bigcup_{x \in X} N_{x} \neq{ }^{\omega} \omega$. Choose an $f \in \omega_{\omega} \omega \backslash \bigcup_{x \in X} N_{x}$. Then the sequence $\left(U_{f(n)}^{n}: n<\infty\right)$ witnesses the Rothberger property of $X$ for $\left(\mathcal{U}_{n}: n<\infty\right)$.

$1 \Rightarrow 2$ : Note that 1 implies that every set of reals of cardinality $\kappa$ has the Rothberger property. Use the fact that $\operatorname{cov}\left(\mathcal{M}_{\mathbb{R}}\right)$ is the minimal cardinality of a set of reals that does not have the Rothberger property ([16, Theorem 5]).

Since $\operatorname{cov}\left(\mathcal{M}_{\mathbb{R}}\right)$ is the least $\kappa$ for which $\mathrm{MA}_{\kappa}$ (countable) is false, we have

COROLlary 30. MA(countable) is equivalent to the statement that every Lindelöf space of cardinality less than $2^{\aleph_{0}}$ has the Rothberger property.

Cardinals forbidden to support points $G_{\delta}$ Rothberger topologies. Another application of ideas in the proof of Theorem 24 gives:

TheOREM 31. If Lebesgue measure can be extended to a countably additive measure on any collection of $2^{\aleph_{0}}$ subsets of the real line, then there is no points $\mathrm{G}_{\delta}$ Rothberger space of cardinality $2^{\aleph_{0}}$.

Proof. Suppose $X$ is a Lindelöf space of cardinality $2^{\aleph_{0}}$ with all points $\mathrm{G}_{\delta}$. We may assume that as a set, $X$ is the closed unit interval. For each $x \in X$ choose a sequence $\left(U_{n}(x): n<\infty\right)$ of neighborhoods with $\{x\}=$ $\bigcap_{n<\infty} U_{n}(x)$ and $U_{n+1}(x) \subseteq U_{n}(x)$ for all $n$. Then $\left\{U_{n}(x): n<\infty, x \in X\right\}$ is a family of $2^{\aleph_{0}}$ subsets of $X$. Let $\mu$ be a countably additive extension of Lebesgue measure which also measures each $U_{n}(x)$.

For each $m$, choose for each $x$ an $n(m, x)$ such that $\mu\left(U_{n(m, x)}(x)\right)<$ $1 / 2^{m+2}$. Then

$$
\mathcal{U}_{m}=\left\{U_{n(m, x)}(x): x \in X\right\}
$$

is an open cover of $X$. For the sequence $\left(\mathcal{U}_{m}: m<\infty\right)$ of open covers of $X$, consider any sequence $\left(U_{m}: m<\infty\right)$ with $U_{m} \in \mathcal{U}_{m}$ for each $m$. Then 
$\mu\left(U_{m}\right)<1 / 2^{m+2}$ for each $m$, and so

$$
\mu\left(\bigcup_{m<\infty} U_{m}\right) \leq \sum_{m=1}^{\infty} \frac{1}{2^{m+2}}<\frac{1}{2}<\mu(X) .
$$

But then the sequence $\left(U_{m}: m<\infty\right)$ does not cover $X$. It follows that $X$ is not Rothberger.

T. Carlson proved that the consistency of the measure extension hypothesis of Theorem 31 implies the consistency of the existence of a weakly compact cardinal, and Carlson and Prikry also derived the consistency of this measure extension hypothesis from the consistency of the existence of a weakly compact cardinal. See Section 6 of [9] in this regard. Thus:

COROLlary 32. If it is consistent that there is a weakly compact cardinal, then it is consistent that there is no points $\mathrm{G}_{\delta}$ Rothberger space of cardinality $2^{\aleph_{0}}$.

By the results of [9], it is for example consistent that $2^{\aleph_{0}}=\aleph_{2}$, and for any family $\mathcal{F}$ of $\aleph_{1}$ subsets of the real line, Lebesgue measure can be extended to a countably additive measure that measures also each element of $\mathcal{F}$. This, however, does not imply that there are no points $\mathrm{G}_{\delta}$ Rothberger spaces of size $\aleph_{1}$, as demonstrated by Example 2 of Section 4.

Carlson and Prikry derived the consistency of the measure extension hypothesis by starting with a model containing a weakly compact cardinal $\kappa$ and then adding $\kappa$ random reals. This leads to an alternative proof of Shelah's result [45] that no weakly compact cardinal can be topologized as a points $\mathrm{G}_{\delta}$ Lindelöf space. First, observe:

COROLlaRY 33. There is no points $\mathrm{G}_{\delta}$ Rothberger topology on a weakly compact cardinal.

Proof. For suppose $X$ is a set of cardinality a weakly compact cardinal, and that $X$ carries a topology in which its points are $\mathrm{G}_{\delta}$, and it is a Rothberger space. Add $|X|$ random reals. By Theorem $15, X$ is still a Rothberger space with points $G_{\delta}$ in the generic extension. But the generic extension is Carlson and Prikry's model for $|X|=2^{\aleph_{0}}$ and the extendibility of Lebesgue measure to any family of $2^{\aleph_{0}}$ subsets of the real line. Then Theorem 31 gives the contradiction that $X$ is not a points $\mathrm{G}_{\delta}$ Rothberger space.

COROLlary 34 (Shelah). There is no points $\mathrm{G}_{\delta}$ Lindelöf topology on a weakly compact cardinal.

Proof. Suppose on the contrary that $\kappa$ is a weakly compact cardinal carrying a points $G_{\delta}$ Lindelöf topology. Add $\aleph_{1}$ Cohen reals. The weakly compact cardinal $\kappa$ is still weakly compact, but by Theorem 11 it now carries a points $\mathrm{G}_{\delta}$ Rothberger topology, contradicting Corollary 33 . 
Continuing the theme that we can prove for Rothberger spaces what we would like to prove for Lindelöf spaces, we obtain in Theorem 35 for Rothberger spaces results discussed but not achieved for Lindelöf spaces in [5]. For $\lambda<\kappa$ infinite regular cardinal numbers let $\mathbf{L v}(\kappa, \lambda)$ be the partially ordered set whose elements are functions $p$ such that $|p|<\lambda$, $\operatorname{dom}(p) \subseteq \kappa \times \lambda$ and for all $(\alpha, \xi) \in \operatorname{dom}(p), p(\alpha, \xi) \in \alpha$; elements $p$ and $q$ are ordered by $p \leq q$ if, and only if, $q \subseteq p$. It is well-known that when $\kappa$ is strongly inaccessible, each antichain of $\mathbf{L v}(\kappa, \lambda)$ is of cardinality less than $\kappa$. Also, as $\lambda$ is regular, $\mathbf{L v}(\kappa, \lambda)$ is $\lambda$-closed. It is also well-known that in the generic extension obtained by forcing with $\mathbf{L v}(\kappa, \lambda), \kappa$ is a cardinal number, but is the successor of $\lambda$. In Theorem 35 the phrase "Lévy-collapse ... to $\omega_{2}$ with countable conditions" means "Force with $\mathbf{L v}\left(\kappa, \omega_{1}\right)$ where $\kappa$ is supercompact (measurable)".

The argument uses standard reflection methods (see e.g. [14]). Specific properties of supercompactness of a cardinal are at the core of these arguments, and are now recalled for the reader's convenience: A cardinal $\kappa$ is supercompact if there is for each cardinal $\lambda \geq \kappa$ an elementary embedding $i: V \rightarrow M$ from the set-theoretic universe $V$ into a transitive class $M$ such that

- for each $\xi<\kappa, i(\xi)=\xi$, but $i(\kappa)>\lambda$,

- ${ }^{\lambda} M \subseteq M$.

Here ${ }^{\lambda} M$ denotes the class of sequences of length $\lambda$, where the terms of the sequences are elements of $M$. It follows by transfinite induction that for all $\alpha \leq \lambda$ we have $M_{\alpha}=V_{\alpha}$-that is, the cumulative hierarchy as computed in $V$ and in $M$ coincides at least up to $\lambda$.

TheOREM 35. Lévy-collapse a supercompact [measurable] cardinal to $\omega_{2}$ with countable conditions. Then every Rothberger $\left(\mathrm{T}_{2}\right)$ space of character $\leq \aleph_{1}$ includes a Rothberger subspace of size $\leq \aleph_{1}$.

Proof. First, the supercompact version. Let $\kappa$ be a supercompact cardinal in the ground model. Let $G$ be $\mathbf{L v}\left(\kappa, \omega_{1}\right)$-generic over $V$. In $V[G]$, let $X$ be a Rothberger space of character $\leq \aleph_{1}$ and cardinality larger than $\aleph_{1}$. Let $\alpha$ be an initial ordinal such that $X$, all open covers of $X$, and all sequences of open covers of $X$, as well as $\mathbf{L v}\left(\kappa, \omega_{1}\right)$ and any of its antichains are members of $V_{\alpha}[G]$. Choose a regular cardinal $\lambda>2^{\alpha}$. In $V[G]$ let $\mu$ be the initial ordinal corresponding to $|X|$. Then $\mu$ is also a cardinal in $V$, and in $V, \kappa \leq \mu<\alpha<\lambda$.

By supercompactness of $\kappa$ fix an elementary embedding $i: V \rightarrow M$ with $i(\kappa)>\lambda$ and ${ }^{\lambda} M \subseteq M$. Since for each $\beta \leq \lambda$ we have $M_{\beta}=V_{\beta}$ it follows that $\mathbf{L v}\left(\kappa, \omega_{1}\right)$ and all $V$-antichains of it are elements of $M$. Thus $G \subseteq$ $\mathbf{L v}\left(\kappa, \omega_{1}\right)$ is $\mathbf{L v}\left(\kappa, \omega_{1}\right)$-generic over $M$ if, and only if, it is over $V$. Moreover, 
$V_{\beta}[G]=M_{\beta}[G]$ for all $\beta \leq \lambda$. It follows that in fact $X \in M[G]$. Moreover, any family of open covers for $X$ in $V[G]$ is also in $M[G]$. Thus, $M[G] \models$ " $X$ is a Rothberger space of cardinality $>\aleph_{1}$ and character $\leq \aleph_{1}$ ".

Since $\mathbf{L v}\left(\kappa, \omega_{1}\right)$ has no antichain of cardinality $\kappa$, there is an $i\left(\mathbf{L v}\left(\kappa, \omega_{1}\right)\right)$ generic (over $M$ ) filter $G^{*}$ such that $p \in G$ implies $i(p) \in G^{*}$ (see Proposition 2.2 of [14]). But then $i$ extends to an elementary embedding $j: V[G] \rightarrow$ $M\left[G^{*}\right]$ (see Proposition 2.1 of [14]).

Until further notice we now work in $M\left[G^{*}\right]$. The equality $i\left(\mathbf{L v}\left(\kappa, \omega_{1}\right)\right)=$ $\mathbf{L v}\left(i(\kappa), \omega_{1}\right)=\mathbf{L v}\left(\kappa, \omega_{1}\right) \times \mathbf{L v}\left(i(\kappa) \backslash \kappa, \omega_{1}\right)$ implies that $M\left[G^{*}\right]$ is of the form $M[G][H]$ where $H$ is $\mathbf{L v}\left(i(\kappa) \backslash \kappa, \omega_{1}\right)$-generic over $M[G]$. By Theorem 21, Rothberger is preserved by countably closed forcing, giving $M\left[G^{*}\right] \models$ " $X$ is a Rothberger space".

The bijection $j\lceil X$ from $X$ to $j[X]$ induces a homeomorphic topology, say $\mathcal{T}$, on $j[X]$ : Put a subset $U$ of $j[X]$ in $\mathcal{T}$ if its inverse image under $j$ is an open subset of $X$. Then $(j[X], \mathcal{T})$ is a Rothberger space.

The subset $j[X]$ of $j(X)$ inherits a topology from $j(X)$, say $\mathcal{S}$. Compare the two spaces $(j[X], \mathcal{T})$ and $(j[X], \mathcal{S})$. First note that $\mathcal{S}$ contains sets of the form $j(U) \cap j[X]$, where $U \subseteq X$ is open in $X$. Conceivably, $\mathcal{S}$ may contain also other sets. Since $j(U) \cap j[X]=j[U]$ (see Lemma 2.0 of [14]) is an element of the topology $\mathcal{T}$ on $j[X]$, we have $\mathcal{T} \subseteq \mathcal{S}$. The character restriction ensures that $\mathcal{S}=\mathcal{T}$ (see the argument at the top of p. 45 of [14]). Thus, $j[X]$ is a Rothberger subspace of $j(X)$.

Since $j$ is an elementary embedding, $j(X)$ is a Rothberger space with character $\leq \aleph_{1}$ and cardinality $j(\mu)$. Since $j[X]$ is an uncountable subset of $j(X)$, we conclude that in $M\left[G^{*}\right]$ the statement

" $j[X] \subseteq j(X)$ is an uncountable Rothberger subspace of $j(X)$ " as well as the statement " $j(\kappa)=\aleph_{2}$ and $|\mu|=\aleph_{1}$ " are true. This implies:

- $M\left[G^{*}\right] \models " j(X)$ has a Rothberger subspace of cardinality $\aleph_{1}$ ".

This concludes working in $M\left[G^{*}\right]$. Since $j\left(\aleph_{1}\right)=\aleph_{1}$ and $j$ is an elementary embedding of $V[G]$ into $M\left[G^{*}\right]$, in $V[G]$ it is true that $X$ has a Rothberger subspace of cardinality $\aleph_{1}$. This concludes the proof for the supercompact case.

For the measurable version, note that a Lindelöf $T_{2}$ space of character $\leq \aleph_{1}$ has cardinality $\leq 2^{\aleph_{1}}$. All the cardinals below the measurable $\kappa$ will have their power sets collapsed, so $2^{\aleph_{1}}=\aleph_{2}$ (the former measurable) in the extension. Measurability yields a nontrivial elementary embedding moving $\kappa$, which again can be extended to a generic elementary embedding, whence we proceed as in the supercompact case.

In [6] Baumgartner and van Douwen introduced the notion of a weakly measurable cardinal: A cardinal number $\kappa$ is said to be weakly measurable 
if there is a sequence $\left(\mathcal{F}_{n}: n<\infty\right)$ of nonprincipal ultrafilters on $\kappa$ such that $\bigcap_{n<\infty} \mathcal{F}_{n}$ is a $\kappa$-complete filter on $\kappa$. It is known that the least such $\kappa$, if not measurable, is strictly less than $2^{\aleph_{0}}$ and that $\kappa \neq \operatorname{cof}\left(2^{\aleph_{0}}\right)$. It is also known that the existence of a measurable cardinal is equiconsistent with the existence of a weakly measurable cardinal less than $2^{\aleph_{0}}$. The model given in Theorem 5.14 of [ $[$ ] for obtaining the consistency of a weakly measurable cardinal below $2^{\aleph_{0}}$ from the consistency of the existence of a measurable cardinal is as follows: Let $\kappa$ be a measurable cardinal in a model satisfying GCH. Let $\lambda>\kappa$ be a cardinal with cofinality not $\kappa$ and not $\omega$. Forcing with $\mathbb{P}(\lambda)$ (the partially ordered set for adding $\lambda$ Cohen reals) produces a model in which $\kappa<\lambda=2^{\aleph_{0}}$ is weakly measurable. Thus, in some sense, weakly measurable cardinals are the Cohen analogue of real-valued measurable cardinals. In this model the set of Cohen reals is a Lusin set and thus has the Rothberger property. This shows that weakly measurable cardinals are not provably upper bounds on the possible cardinality of points $\mathrm{G}_{\delta}$ Rothberger spaces. Moreover, since every uncountable subset of a Lusin set is a Lusin set, every subset of this set of Cohen reals has the Rothberger property, and thus there are points $\mathrm{G}_{\delta}$ Rothberger spaces of all cardinalities less than or equal to $\lambda$ in this model.

3. The Gerlits-Nagy property. Gerlits and Nagy [18] used the symbol $*$ to denote one of the several covering properties they introduced. We shall call $*$ the Gerlits-Nagy property, and define it later below. Though the Gerlits-Nagy property is formally stronger than the Rothberger property the relationship between these two properties is somewhat complicated. Often ZFC examples of Rothberger spaces are also Gerlits-Nagy spaces. Some reasons for this phenomenon will be discussed below. An examination of Gerlits-Nagy spaces requires examining the Hurewicz and Menger properties which we introduce now.

The symbol $\mathrm{S}_{\text {fin }}^{\alpha}(\mathcal{A}, \mathcal{B})$ denotes the statement:

For each sequence $\left(A_{\gamma}: \gamma<\alpha\right)$ of elements of $\mathcal{A}$, there is a sequence $\left(B_{\gamma}: \gamma<\alpha\right)$ of finite sets such that for each $\gamma$ we have $B_{\gamma} \subseteq A_{\gamma}$, and $\bigcup_{\gamma<\alpha} B_{\gamma} \in \mathcal{B}$.

The corresponding game, denoted $\mathrm{G}_{\text {fin }}^{\alpha}(\mathcal{A}, \mathcal{B})$, is played as follows: Players ONE and TWO play $\alpha$ innings. In the $\gamma$ th inning ONE chooses an $O_{\gamma} \in \mathcal{A}$, and TWO responds with a finite set $T_{\gamma} \subseteq O_{\gamma}$. A play

$$
O_{0}, T_{0}, \ldots, O_{\gamma}, T_{\gamma}, \ldots
$$

is won by TWO if $\bigcup_{\gamma<\alpha} T_{\gamma} \in \mathcal{B}$; else, ONE wins.

$\mathrm{S}_{\text {fin }}^{\omega}(\mathcal{O}, \mathcal{O})$ is known as the Menger property. W. Hurewicz [21] introduced this property and showed that in metrizable spaces it is equivalent to a 
basis property introduced by K. Menger [34. Hurewicz also showed that a space has the property $\mathrm{S}_{\text {fin }}^{\omega}(\mathcal{O}, \mathcal{O})$ if, and only if, ONE has no winning strategy in the game $\mathrm{G}_{\text {fin }}^{\omega}(\mathcal{O}, \mathcal{O})$. An exposition of Hurewicz's result is given in Theorem 13 of [42]. Clearly, the Rothberger property implies the Menger property.

Hurewicz 21] also introduced a property stronger than the Menger property, called the Hurewicz property. The Hurewicz property is defined as follows: For each sequence $\left(\mathcal{U}_{n}: n<\omega\right)$ of open covers of $X$ there is a sequence $\left(\mathcal{V}_{n}: n<\omega\right)$ of finite sets such that for each $n, \mathcal{V}_{n} \subseteq \mathcal{U}_{n}$, and such that for each $x \in X$, for all but finitely many $n, x \in \bigcup \mathcal{V}_{n}$. The Hurewicz property can also be described as follows:

Call an open cover $\mathcal{U}$ of a space large if each element of the space is contained in infinitely many members of $\mathcal{U}$. The symbol $\Lambda$ denotes the collection of large covers of a space. One can show that $S_{\text {fin }}^{\omega}(\mathcal{O}, \mathcal{O})$ is equivalent to $\mathrm{S}_{\text {fin }}^{\omega}(\Lambda, \Lambda)$.

A large cover $\mathcal{U}$ of a space $X$ is groupable if there is a partition $\mathcal{U}=$ $\bigcup_{n<\infty} \mathcal{U}_{n}$ such that each $\mathcal{U}_{n}$ is finite, for $m \neq n$ we have $\mathcal{U}_{m} \cap \mathcal{U}_{n}=\emptyset$, and for each $x \in X$, for all but finitely many $n, x \in \bigcup \mathcal{U}_{n}$.

By Theorem 12 of [30] a Lindelöf space has the Hurewicz property if, and only if, it has the Menger property and each countable large cover is groupable. $\sigma$-compactness implies the Hurewicz property, and the Hurewicz property implies the Menger property. Menger conjectured that in separable metric spaces the Menger property implies $\sigma$-compactness; Hurewicz conjectured that in separable metric spaces the Hurewicz property implies $\sigma$-compactness. It is known in ZFC that even in the class of separable metric spaces none of the above implications is reversible: In [11] it is shown that the Menger property does not imply the Hurewicz property; in [27] it is shown that the Hurewicz property does not imply $\sigma$-compactness. For more on these implications, see [58]. Telgársky [53] proved that if $X$ is a separable metric space and TWO has a winning strategy in the game $\mathrm{G}_{\text {fin }}^{\omega}(\mathcal{O}, \mathcal{O})$, then $X$ is $\sigma$-compact (the converse is evidently true).

In Theorems 14 and 19 of [37] it is shown that the Gerlits-Nagy property is equivalent to the Hurewicz property plus the Rothberger property. We take this characterization as our definition of the Gerlits-Nagy property.

Cohen reals and the Hurewicz property. We have already seen that Cohen reals do not preserve not Rothberger. Thus, Cohen reals do not preserve not Menger. The situation for the Hurewicz property, and thus the Gerlits-Nagy property, is different. In the proof of Theorem 37 we use the following well-known fact: 
Lemma 36. If $\mathcal{F} \subseteq{ }^{\omega} \omega$ is unbounded in the eventual domination order and $\kappa>0$ is a cardinal number then

$$
\mathbf{1}_{\mathbb{P}(\kappa)} \|- \text { “泣 is unbounded". }
$$

Though we have not found a direct reference (which undoubtedly exists), Lemma 36 can be deduced from [50. Lemma 3.1], together with the fact that for any $\mathbb{P}(\kappa)$-name $\dot{f}$ there is a countable set $S \subseteq \kappa$ with $\dot{f}$ in fact a $\mathbb{P}(S)$-name.

TheOREM 37. Let $\kappa>0$ be a cardinal number and let $X$ be a Lindelöf space. If $X$ does not have the Hurewicz property then

$$
\mathbf{1}_{\mathbb{P}(\kappa)} \|- \text { "X does not have the Hurewicz property". }
$$

Proof. Let $X$ be a Lindelöf space which is not Hurewicz. Let the sequence $\left(\mathcal{U}_{n}: n<\omega\right)$ of open covers of $X$ witness that $X$ is not Hurewicz. Since $X$ is Lindelöf, we may assume each $\mathcal{U}_{n}$ is countable and has an enumeration $\left(U_{k}^{n}: k<\omega\right)$ such that for all $n$ and $k, U_{k}^{n} \subseteq U_{k+1}^{n}$. We shall show that

$$
\left.\mathbf{1}_{\mathbb{P}(\kappa)} \|- \text { "( } \check{\mathcal{U}}_{n}: n \in \check{\omega}\right) \text { witnesses that } \check{X} \text { is not Hurewicz". }
$$

Suppose the contrary and choose $p \in \mathbb{P}(\kappa)$ such that $p \|_{-}$" $\check{X}$ is Hurewicz". For each $x \in X$ define a function $f_{x}: \omega \rightarrow \omega$ such that for each $n$, $f_{x}(n)=\min \left\{k:(\forall m \geq k)\left(x \in U_{m}^{n}\right)\right\}$. Note that the particular sequence of open covers of $X$ witness that $X$ is not Hurewicz if, and only if, the associated family of functions $\left\{f_{x}: x \in X\right\}$ is unbounded. Since $X$ is not Hurewicz, $\left\{f_{x}: x \in X\right\}$ is unbounded in ${ }^{\omega} \omega$.

However, $p \|-$ " $\left(\exists \tau \in{ }^{\omega} \omega\right)(\forall x \in \check{X})\left(f_{x} \prec \tau\right)$ " $\left({ }^{3}\right)$, Choose a $\mathbb{P}(\kappa)$ name $\dot{f}$ such that $p \|_{-}$" $(\forall x \in \check{X})\left(f_{x} \prec \dot{f}\right)$ ". Then choose a countable subset $C$ of $\kappa$ such that $\dot{f}$ is a $\mathbb{P}(C)$-name and $p \in \mathbb{P}(C)$. This gives a contradiction: $\mathbf{1}_{\mathbb{P}(C)} \|-"\left\{f_{x}: x \in \check{X}\right\}$ is unbounded" (by Lemma 36) while $p \|-"\left\{f_{x}\right.$ : $x \in \check{X}\}$ is bounded".

The referee pointed out that the Cohen reals poset $\mathbb{P}(\kappa)$ does not preserve the Hurewicz property, which is most efficiently seen as follows: If $X$ is a codense subset of a complete metric space $M$ and if $X$ has the Hurewicz property, then $X$ is a meager subset of $M$ (see the proof of Theorem 5.5 of [27]). But it is well-known that upon forcing with $\mathbb{P}(\omega)$, the set of groundmodel reals is a codense subset of the set of real numbers of the extension, but is not a meager subset of the reals of the extension (see Theorem 3.2 of [50]). To now see this is the case for $\mathbb{P}(\kappa)$ also for uncountable $\kappa$, note: A $\mathbb{P}(\kappa)$-name for a comeager Borel set disjoint from the ground-model reals is, for some countable subset $C$ of $\kappa$, a $\mathbb{P}(C)$-name.

$\left({ }^{3}\right) f \prec g$ denotes that for all but finitely many $n, f(n)<g(n)$. 
There is a limited class of Hurewicz spaces for which we could prove that Hurewicz is preserved by Cohen real forcing. This class of Hurewicz spaces has appeared under different names in the study of small sets of reals. In [39] these are called "property B", while in [4] they are called "sets in $\mathcal{H}$ ". Call a Hurewicz space $X$ strongly Hurewicz if for each Borel function $F: X \rightarrow{ }^{\omega} \omega$ there is a function $g \in{ }^{\omega} \omega$ such that for each $x \in X$, for all but finitely many $n, F(x)(n)<g(n)$. Some properties of this class of Hurewicz spaces can be found in [44, such as that such subsets of the real line are zero-dimensional and that Sierpiński sets are strongly Hurewicz.

Recall the notion of an $n$-dowment, defined as follows: Fix a positive integer $n$. A family $\mathcal{L}$ of finite subsets of $\mathrm{Fn}(\kappa, 2)$ is said to be an $n$-dowment if for each maximal antichain $A \subseteq \mathrm{Fn}(\kappa, 2)$ there is an $L \in \mathcal{L}$ with $L \subseteq A$, and for each $p \in \operatorname{Fn}(\kappa, 2)$ with domain of cardinality $n$ and for any $L_{1}, \ldots, L_{n} \in \mathcal{L}$ there are $q_{i} \in L_{i}, i \leq n$ such that $\left\{q_{i}: i \leq n\right\} \cup\{p\}$ have a common extension in $\mathrm{Fn}(\kappa, 2)$. For each $\kappa$ and each $n$ there is an $n$-dowment-see Lemma 1.1 of [14].

Also recall that the Hurewicz game on a space $X$ is played as follows: ONE and TWO play an inning for each positive integer $n$. In the $n$th inning ONE first chooses an open cover $O_{n}$ for $X$, and then TWO responds with a finite set $T_{n} \subseteq O_{n}$. A play $O_{1}, T_{1}, \ldots, O_{n}, T_{n}, \ldots$ is won by TWO if for each $x \in X$, for all but finitely many $n, x \in \bigcup T_{n}$; else, ONE wins. The game was introduced in 42 where it was shown in Theorem 27 that $X$ is a Hurewicz space if, and only if, ONE has no winning strategy in the Hurewicz game on $X$.

For the special case of a separable metric space we use the following alternative characterization of the Hurewicz property of subsets of $\mathbb{H}=$ $[0,1]^{\mathbb{N}}$, the Hilbert cube $\left({ }^{4}\right)$;

Proposition 38 ([27]). A subspace $X$ of $\mathbb{H}$ has the Hurewicz property if, and only if, for each $\mathrm{G}_{\delta}$-subset $G$ of $\mathbb{H}$ with $X \subseteq G$, there is an $\mathrm{F}_{\sigma}$-subset $F$ of $\mathbb{H}$ with $X \subseteq F \subseteq G$.

Proof. See Theorem 5.7 of [27]: the proof given there is for the real line, but adapts easily to the Hilbert cube.

More generally this argument also applies to characterizing Hurewicz subsets of $[0,1]^{\lambda}$. We shall also need the following observation:

LEMma 39. Let $\lambda$ be an uncountable cardinal number and let $X$ be a Lindelöf subset of $[0,1]^{\lambda}$. For each $\mathrm{G}_{\delta}$-set $G \subseteq[0,1]^{\lambda}$ such that $X \subseteq G$ there is a $\mathrm{G}_{\delta}$-set $H$ and a countable set $C \subseteq \lambda$ such that

$\left({ }^{4}\right)$ This characterization of Hurewicz, suitably reformulated, holds in greater generality: 52 extended it to Lindelöf $\mathrm{T}_{3}$ spaces. 
1. $X \subseteq H \subseteq G$,

2. $H$ is homeomorphic to $\Pi_{C}[H] \times[0,1]^{\lambda \backslash C}$.

Proof. First, consider an open set $U \subseteq[0,1]^{\lambda}$ with $X \subseteq U$. Choose for each $x \in X$ a basic open set $B(U, x)$ of the form $\prod_{\alpha \in \lambda} S_{\alpha} \subseteq U$ where the set $F(U, x)$, defined as

$\left\{\alpha \in \lambda: S_{\alpha}\right.$ is a proper open subset of $[0,1]$ with rational endpoints $\}$, is finite. Then $\{B(U, x): x \in X\}$ is an open cover of $X$ and thus has a countable subset $\left\{B\left(U, x_{n}\right): n<\omega\right\}$ which covers $X$. Then $C_{U}=\bigcup_{n<\omega} F\left(U, x_{n}\right)$ is a countable subset of $\lambda$, and $V=\bigcup_{n<\omega} B\left(U, x_{n}\right)$ is an open set with $X \subseteq V \subseteq U$, and for any countable set $D$ with $C_{U} \subseteq D \subseteq \lambda, V$ is homeomorphic to $\Pi_{D}[V] \times[0,1]^{\lambda \backslash D}$.

Next, let $G$ be a $\mathrm{G}_{\delta}$-set containing $X$, and choose open sets $U_{1} \supseteq U_{2} \supseteq \cdots$ such that $G=\bigcap_{n<\omega} U_{n}$. Applying the preceding remarks consecutively to each $U_{n}$ we find open sets $V_{n}$ and countable sets $C_{n} \subseteq \lambda$ such that for each $n$ we have

1. $U_{n} \supseteq V_{n} \supseteq V_{n+1} \supseteq X$.

2. $C_{n} \subseteq C_{n+1}$.

3. For each countable $D$ with $C_{n} \subseteq D \subseteq \lambda, V_{n}$ is homeomorphic to $\Pi_{D}\left[V_{n}\right] \times[0,1]^{\lambda \backslash D}$.

Finally put $H=\bigcap_{n<\omega} V_{n}$, and $C=\bigcup_{n<\omega} C_{n}$.

The following theorem is used in the proof of Corollary 48 .

THEOREM 40. Let $\kappa>0$ be a cardinal number and let $X$ be a subspace of $\mathbb{R}$. If $X$ is a strong Hurewicz space, then $\mathbf{1}_{\mathbb{P}(\kappa)} \|-$ " $\check{X}$ has the Hurewicz property".

Proof. Since a union of countably many Hurewicz spaces is a Hurewicz space, we may assume that $X$ is a subspace of $[0,1]$. For each positive integer $n$, let $L_{n}$ be an $n$-dowment, as defined above.

Since $X$ is a set of real numbers, it suffices to show that the following statement is true in generic extensions by $\mathbb{P}(\kappa)$ :

For each $\mathrm{G}_{\delta}$-set $G \subseteq[0,1]$ with $X \subseteq G$ there is an $\mathrm{F}_{\sigma^{-} \text {-set } F \text { such that }}$ $X \subseteq F \subseteq G$.

Thus, let a sequence $\left(\dot{G}_{n}: n<\omega\right)$ of $\mathbb{P}(\kappa)$-names be given such that $\mathbf{1}_{\mathbb{P}(\kappa)} \|-"(\forall n)\left(\dot{G}_{n} \subseteq[0,1]\right.$ is open and includes $\check{X}$ and $\left.\dot{G}_{n} \supseteq \dot{G}_{n+1}\right) "$. Then for each $n$ let $\dot{C}_{n}$ be a $\mathbb{P}(\kappa)$-name such that $\mathbf{1}_{\mathbb{P}(\kappa)} \|-"(\forall n)\left(\dot{C}_{n}=[0,1] \backslash \dot{G}_{n}\right.$ is compact and $\left.\check{X} \cap \dot{C}_{n}=\emptyset\right) "$. Then for each $n$ and each $x \in X$, $\mathbf{1}_{\mathbb{P}(\kappa)} \|-"\left(\exists \dot{U}_{n}\right)\left(\exists \dot{V}_{n}\right)\left(\dot{U}_{n}\right.$ and $\dot{V}_{n}$ open, $\overline{\dot{U}_{n}} \cap \overline{\dot{V}_{n}}=\emptyset$ and $\left.x \in \dot{U}_{n}, \dot{C}_{n} \subseteq \dot{V}_{n}\right) "$. 
Choose for each $x \in X$ and each $n<\omega$ a maximal antichain $A\left(x, \dot{C}_{n}\right) \subseteq$ $\mathbb{P}(\kappa)$, and for each $p \in A\left(x, \dot{C}_{n}\right)$ a clopen (in $X$ ) interval $U_{p}\left(x, \dot{C}_{n}\right)$, a $\mathbb{P}(\kappa)$ name $\dot{V}_{p, x}\left(\dot{C}_{n}\right)$ and a finite set $Q_{p, x}\left(\dot{C}_{n}\right)$ of pairs $r<s$ of rational numbers such that with $\dot{I}(r, s)$ the $\mathbb{P}(\kappa)$-name for the open interval $(\check{r}, \check{s}), p$ forces the conjunction of the following three statements:

1) " $\dot{V}_{p, x}\left(\dot{C}_{n}\right)=\bigcup\left\{\dot{I}(r, s):(r, s) \in Q_{p, x}\left(\dot{C}_{n}\right)\right\} "$,

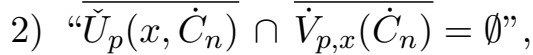

3) " $\dot{C}_{n} \subseteq \dot{V}_{p, x}\left(\dot{C}_{n}\right)$ ".

In the ground model it is true that $U_{p}\left(x, \dot{C}_{n}\right)$ is disjoint from $\bigcup\{(r, s)$ : $\left.(r, s) \in Q_{p, x}\left(\dot{C}_{n}\right)\right\}$, because $\mathbf{1}_{\mathbb{P}(\kappa)} \|-"(r, s)^{\swarrow} \subseteq \dot{I}(r, s)$ ".

Then for each $x, n$ and $k$ choose a finite set $F_{k}\left(x, \dot{C}_{n}\right) \subseteq A\left(x, \dot{C}_{n}\right)$ which is a member of the $k$-dowment $L_{k}$. For fixed $n$ and $k$ define, for each $x$,

4) $U_{k}^{n}(x)=\bigcap\left\{U_{p}\left(x, \dot{C}_{n}\right): p \in F_{k}\left(x, \dot{C}_{n}\right)\right\}$,

5) $\dot{V}_{k, x}^{n}=\bigcap\left\{\dot{V}_{p, x}\left(\dot{C}_{n}\right): p \in F_{k}\left(x, \dot{C}_{n}\right)\right\}$.

In 5) we have defined a $\mathbb{P}(\kappa)$-name. For there is in the ground model the corresponding finite set $Q_{k, x}^{n}$ of pairs $(r, s)$ of rational numbers obtained from the corresponding finite sets $Q_{p, x}\left(\dot{C}_{n}\right), p \in F_{k}\left(x, \dot{C}_{n}\right)$ (by taking the set of intersections of the finitely many rational intervals associated with the sets $\left.Q_{p, x}\left(\dot{C}_{n}\right)\right)$ such that $\dot{V}_{k, x}^{n}=\bigcup\left\{\dot{I}(r, s):(r, s) \in Q_{k, x}^{n}\right\}$. Define in the ground model the set $v_{k, x}^{n}=\bigcup_{(r, s) \in Q_{k, x}^{n}}(r, s)$. Observe that for each $p \in F_{k}\left(x, \dot{C}_{n}\right)$ we have $p \|-" \overline{U_{k}^{n}(x)} \cap \overline{\dot{V}_{k, x}^{n}}=\emptyset "$, and in the ground model we have $\overline{U_{k}^{n}(x)} \cap \overline{v_{k, x}^{n}}=\emptyset$.

Next, for each $n$ and $k$ define $\mathcal{U}_{n, k}=\left\{U_{k}^{n}(x): x \in X\right\}$, a ground-model clopen cover of $X$. For each fixed $n$ define a strategy $\sigma_{n}$ of player ONE of the Hurewicz game on $X$ as follows:

Definition of $\sigma_{n}(\emptyset): \sigma_{n}(\emptyset)=\mathcal{U}_{n, 1}$.

Suppose that TWO responds with the finite set $T_{1}^{n} \subseteq \sigma_{n}(\emptyset)$.

Definition of $\sigma_{n}\left(T_{1}^{n}\right)$ : Fix a finite set $\left\{x_{1}^{n, 1}, \ldots, x_{\ell_{1}^{n}}^{n, 1}\right\} \subseteq X$ with $T_{1}^{n}=$ $\left\{U_{1}^{n}\left(x_{i}^{n, 1}\right): i \leq \ell_{1}^{n}\right\}$. Define $m_{1}^{n}=\max \left\{|p|: p \in \bigcup_{i \leq \ell_{1}^{n}} F_{1}\left(x_{i}^{n, 1}, \dot{C}_{n}\right)\right\}$. Then put

$$
\sigma_{n}\left(T_{1}^{n}\right)=\mathcal{U}_{n, m_{1}^{n}+1}
$$

Suppose that TWO responds with the finite set $T_{2}^{n} \subseteq \sigma_{n}\left(T_{1}^{n}\right)$.

Definition of $\sigma_{n}\left(T_{1}^{n}, T_{2}^{n}\right)$ : Fix a finite set $\left\{x_{1}^{n, 2}, \ldots, x_{\ell_{2}^{n}}^{n, 2}\right\} \subseteq X$ with $T_{2}^{n}=$ $\left\{U_{m_{1}+1}^{n}\left(x_{i}^{n, 2}\right): i \leq \ell_{2}^{n}\right\}$. Define $m_{2}^{n}=\max \left\{|p|: p \in \bigcup_{i \leq \ell_{2}^{n}} F_{m_{1}^{n}+1}\left(x_{i}^{n, 2}, \dot{C}_{n}\right)\right\}$. Then put

$$
\sigma_{n}\left(T_{1}^{n}, T_{2}^{n}\right)=\mathcal{U}_{n, m_{1}^{n}+m_{2}^{n}+1},
$$

and so on. 
Since $X$ has the Hurewicz property, $\sigma_{n}$ is not a winning strategy for ONE. Choose a $\sigma_{n}$-play lost by ONE, and let TWO's sequence of moves in this play be $T_{1}^{n}, T_{2}^{n}, \ldots$ Then for each $x \in X$, for all but finitely many $k$, we have $x \in \bigcup T_{k}^{n}$. For this sequence of moves by TWO fix the symbols $x_{i}^{n, k}$, $\ell_{k}^{n}$ and $m_{k}^{n}$ as above in the definition of the strategy $\sigma_{n}$.

Note that for each $n$ we have: For each $j$, if $p \in F_{m_{1}^{n}+\cdots+m_{j-1}^{n}+1}\left(x_{i}^{n, j}, \dot{C}_{n}\right)$ for some $i \leq \ell_{j}^{n}$, then $p \|-“ \overline{U \check{T}_{j}^{n}} \cap \overline{\dot{V}_{j}^{n}}=\emptyset$ ", where henceforth

6) $\dot{V}_{j}^{n}$ is a $\mathbb{P}(\kappa)$-name for $\dot{V}_{j-1}^{n} \cap \bigcap_{i \leq \ell_{j}^{n}} \dot{V}_{j, x_{i}^{n, j}}^{n}$.

Now consider an arbitrary $p \in \mathbb{P}(\kappa)$ and an arbitrary $n$. Consider any $k$ so large that $|p|<m_{1}^{n}+\cdots+m_{k-1}^{n}+1$. Then for each $x_{i}^{n, k}$ with $i \leq \ell_{k}^{n}$, find a $q_{i} \in F_{m_{1}^{n}+\cdots+m_{k-1}^{n}+1}\left(x_{i}^{n, k}, \dot{C}_{n}\right)$ such that each pair $\left\{q_{i}, p\right\}$ has a common extension, say $r_{i}$. Then $r_{i} \|-$ " $(\forall t \geq k)\left(\overline{\cup \check{T}_{k}^{n}} \cap \overline{\dot{V}_{t}^{n}}=\emptyset\right)$ ". It follows that

$$
p \|-"\left(\exists S_{n} \in[\check{\omega}]^{\aleph_{0}}\right)\left(\forall k \in S_{n}\right)(\forall t \geq k)\left(\overline{\bigcup_{T_{k}^{n}}^{n}} \cap \overline{\dot{V}_{t}^{n}}=\emptyset\right) " .
$$

Since $p$ and $n$ were arbitrary we find that in fact

$$
\mathbf{1}_{\mathbb{P}(\kappa)} \|-“(\forall n)\left(\exists S_{n} \in[\check{\omega}]^{\aleph_{0}}\right)\left(\forall k \in S_{n}\right)(\forall t \geq k)\left(\overline{\bar{U}_{\bar{T}}^{n}} \cap \overline{\dot{V}_{t}^{n}}=\emptyset\right) " .
$$

In the ground model define a function $F: X \rightarrow{ }^{\omega} \omega$ as follows:

$$
F(x)(n)=\min \left\{k:(\forall j \geq k)\left(x \in \bigcup T_{j}^{n}\right)\right\} .
$$

Since for each basic open subset $\left[\left(^{5}\right)\left[\left(n_{1}, \ldots, n_{j}\right)\right]\right.$ of ${ }^{\omega} \omega$ we have

$$
F^{-1}\left[\left[\left(n_{1}, \ldots, n_{j}\right)\right]\right]=\bigcap_{i \leq j}\left(\bigcap_{t \geq n_{i}}\left(\bigcup T_{j}^{i}\right) \backslash \bigcup T_{n_{i}-1}^{i}\right),
$$

a Borel subset of $X$, and thus $F$ is a Borel function. Since $X$ is strongly Hurewicz we fix an increasing $g \in \omega_{\omega} \omega$ such that for each $x \in X$, for all but finitely many $n, F(x)(n)<g(n)$.

Let $H$ be $\mathbb{P}(\kappa)$-generic. In $V[H]$ we do the following:

For each $n$ choose an infinite subset $S_{n}$ of $\omega$ such that for each $k \in S_{n}$, for all $t \geq k, \overline{U T_{k}^{n}} \cap \overline{V_{t}^{n}}=\emptyset$. Then, for each $n$, choose $f(n) \in S_{n}$ with $f(n)>g(n)$.

It follows that

7) For each $x$, for all but finitely many $n, x \in \bigcup T_{f(n)}^{n}$.

8) For each $n$, for all $t \geq f(n), \overline{U T_{f(n)}^{n}} \cap \overline{V_{t}^{n}}=\emptyset$.

For each $k$ put $F_{k}=\bigcap_{n \geq k} \overline{\cup T_{f(n)}^{n}}$, a closed set. By 3), 5),6) and 8) above, $F_{k}$ is disjoint from $\bigcup_{n \geq k} C_{n}$, and thus included in $\bigcap_{n \geq k} G_{n}$. Since $G_{i} \supseteq G_{j}$ for $i<j$ we find that $\bar{F}_{k}$ is an $\mathrm{F}_{\sigma}$-set contained in $G$.

$\left({ }^{5}\right)$ The symbol $\left[\left(n_{1}, \ldots, n_{j}\right)\right]$ denotes the set $\left\{f \in{ }^{\omega} \omega:(\forall i \leq j)\left(f(i)=n_{i}\right)\right\}$. 
By 7), $X \subseteq \bigcup_{k<\omega} F_{k}$, where the latter is an $\mathrm{F}_{\sigma}$-set. It follows that in

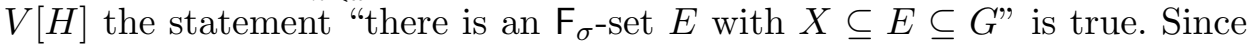
$H$ was an arbitrary $\mathbb{P}(\kappa)$-generic filter, we have now proven

$\mathbf{1}_{\mathbb{P}(\kappa)} \|-$ " $\check{X}$ is a Hurewicz space in the topology inherited from $\dot{\mathbb{R}}$ ".

But since in the generic extension the topology of $X$ generated by the ground-model topology of $X$ is a subset of the topology $X$ inherits from the real line of the generic extension, it finally follows that $\left({ }^{6}\right)$

$$
\mathbf{1}_{\mathbb{P}(\kappa)} \|- \text { " } \check{X} \text { is a Hurewicz space". }
$$

Problem 6. Does Cohen real forcing preserve the strong Hurewicz property?

Adding enough Cohen reals converts ground-model strongly Hurewicz spaces to Gerlits-Nagy spaces in the generic extension:

COROllary 41. Let $\kappa$ be an uncountable cardinal. For a space $X \subseteq \mathbb{R}$, if $X$ has the strong Hurewicz property, then $\mathbf{1}_{\mathbb{P}(\kappa)} \|-$ " $\check{X}$ has the Gerlits-Nagy property".

Proof. The Hurewicz property implies the Lindelöf property. Now apply Theorems 11 and 40 to conclude that a ground-model strongly Hurewicz subspace of $\mathbb{R}$ is converted to a Rothberger space which still has the Hurewicz property.

\section{Random reals and the Hurewicz and Menger properties}

THEOREM 42. Let $\kappa$ be a cardinal number. If $X$ is a Lindelöf space, the following are equivalent:

1. $X$ has the Menger property.

2. $\mathbf{1}_{\mathbb{B}(\kappa)} \|-$ "X has the Menger property".

Proof. Suppose that $X$ is a Lindelöf space.

$2 \Rightarrow 1$ : Suppose that for some positive element $b$ of $\mathbb{B}(\kappa)$,

$$
b \|- \text { " } \check{X} \text { has the Menger property } \mathrm{S}_{\text {fin }}^{\omega}(\mathcal{O}, \mathcal{O}) \text { ". }
$$

Let $\left(\mathcal{U}_{n}: n<\omega\right)$ be a ground-model sequence of open covers of $X$. We may assume each $\mathcal{U}_{n}$ is countable and enumerate it as $\left(U_{m}^{n}: m<\omega\right)$. Then

$$
b \|- \text { " }\left(\check{\mathcal{U}}_{n}: n<\omega\right) \text { is a sequence of open covers of } \check{X} \text { " }
$$

and thus

$$
b \|-"\left(\exists \phi \in{ }^{\omega} \omega\right)(\forall x \in \check{X})\left(\exists_{n}^{\infty}\right)\left(x \in \bigcup_{j \leq \phi(n)} U_{j}^{n}\right) " .
$$

$\left({ }^{6}\right)$ In general, if $\tau_{1} \subseteq \tau_{2}$ are topologies on a space $X$ and $\left(X, \tau_{2}\right)$ has the Lindelöf, Rothberger, Hurewicz or Menger property, then so does $\left(X, \tau_{1}\right)$. 
Now it is well-known that there is an $f \in{ }^{\omega} \omega$ such that

$$
b \|-“\left(\forall_{n}^{\infty}\right)(\phi(n)<f(n)) " .
$$

Fix such an $f$. Then

$$
b \|- \text { " }(\forall x \in \check{X})\left(\exists_{n}^{\infty}\right)\left(x \in \bigcup_{j \leq f(n)} U_{j}^{n}\right) " .
$$

But all parameters in this last sentence are from the ground model, thus the sentence is true in the ground model, and we find an $f \in{ }^{\omega} \omega$ such that setting $\mathcal{V}_{n}=\left\{U_{j}^{n}: j \leq f(n)\right\}$, the sequence of $\mathcal{V}_{n}$ 's cover $X$. We conclude that $X$ has the Menger property in the ground model.

$1 \Rightarrow 2$ : Consider a $\mathbb{B}(\kappa)$-name $\left(\mathcal{U}_{n}: n<\infty\right)$ such that

$$
\mathbf{1}_{\mathbb{B}(\kappa)} \|-"\left(\mathcal{U}_{n}: n<\infty\right) \text { is a sequence of open covers of } \check{X} " \text {. }
$$

Since the ground-model topology is a basis for the topology of $X$ in the generic extension, we may assume that

$\mathbf{1}_{\mathbb{B}(\kappa)} \|-$ "Each $\mathcal{U}_{n}$ consists of sets open in the ground-model topology".

For each $x \in X$ and each $n$, choose a maximal antichain $A_{x}^{n} \subseteq \mathbb{B}(\kappa)$ such that whenever $b \in A_{x}^{n}$ then there is a neighborhood $V_{b}^{n}(x)$ of $x$ such that $b \|-$ " $\left(\exists U \in \mathcal{U}_{n}\right)\left(V_{b}^{n}(x) \subseteq U\right)$ ". Then choose a finite set $L_{x}^{n} \subseteq A_{x}^{n}$ such that $\mu\left(\bigcup L_{x}^{n}\right) \geq 1-(1 / 2)^{n}$. Then put

$$
W_{n}(x)=\bigcap\left\{V_{b}^{n}(x): b \in L_{x}^{n}\right\} .
$$

The set $\mathcal{G}_{n}:=\left\{W_{n}(x): x \in X\right\}$ is a ground-model open cover of $X$.

Claim 1. $(\forall x \in X)(\forall b \in \mathbb{B}(\kappa))(\exists m<\infty)(\forall n \geq m)\left(\left(\exists V \in \mathcal{G}_{n}\right)(x \in V)\right.$ $\left.\Rightarrow(\exists r \leq b)\left(r \|-"\left(\exists U \in \mathcal{U}_{n}\right)(V \subseteq U) "\right)\right)$.

To see this, consider an $x \in X$ and a $b \in \mathbb{B}(\kappa)$. Choose $m$ so large that $1 / 2^{m}<\mu(b)$. Consider any $n \geq m$. There are $V \in \mathcal{G}_{n}$ with $x \in V$. Fix such a $V$, say $V=W_{n}(y)$ for some $y \in X$. Then

$$
\mu\left(\left\|\left(\exists U \in \mathcal{U}_{n}\right)(V \subseteq U)\right\|\right) \geq 1-(1 / 2)^{n} .
$$

Set $r=b \cap\left\|\left(\exists U \in \mathcal{U}_{n}\right)(V \subseteq U)\right\|$. This completes the proof of Claim 1 .

Observe that the $m$ in Claim 1 can be replaced with any larger value. Since $X$ has the Menger property in the ground model, choose there for each $n$ a finite set $\mathcal{H}_{n} \subseteq \mathcal{G}_{n}$ such that

$$
(\forall x \in X)\left(\exists_{n}^{\infty}\right)\left(x \in \bigcup \mathcal{H}_{n}\right) .
$$

Claim 2. $D_{x}^{m}=\left\{b \in \mathbb{B}(\kappa):(\exists n>m)\left(\exists V \in \mathcal{H}_{n}\right)(x \in V\right.$ and $b \|$ " $\left(\exists U \in \check{\mathcal{U}}_{n}\right)(V \subseteq U)$ ") $\}$ is dense in $\mathbb{B}(\kappa)$.

To see this, consider $x \in X$ and $m<\infty$. Consider any $b \in \mathbb{B}(\kappa)$. Choose by Claim 1 an $M>m$ such that for any $n \geq M$, if there is a $V \in \mathcal{G}_{n}$ with $x \in V$, then there is an $r \leq b$ such that $r \|-"\left(\exists U \in \mathcal{U}_{n}\right)(V \subseteq U)$ ". Then 
choose $n>M$ with $x \in \mathcal{H}_{n}$. Apply Claim 1 to obtain a member $q$ of $D_{x}^{m}$ with $q<b$. This completes the proof of Claim 2 .

Now let $G$ be a $\mathbb{B}(\kappa)$-generic filter. For each $m$ and $x \in X$ choose a $b(m, x) \in D_{x}^{m} \cap G$. Then choose $n(m, x)>m$ and $H(m, x) \in \mathcal{H}_{n(m, x)}$ with $x \in H(m, x)$. Now $b(m, x) \|-"\left(\exists U \in \mathcal{U}_{n(m, x)}\right)(H(m, x) \subseteq U)$ ", and thus this forced sentence is true in the generic extension by $G$. In the generic extension choose for each such $n$, and for each $V \in \mathcal{H}_{n}$ for which it is possible, a $U_{V} \in \mathcal{U}_{n}$ with $V \subseteq U_{V}$, and let $\mathcal{J}_{n}$ be the set of such chosen $U_{V}$ 's. Then in the generic extension, for each $n, \mathcal{J}_{n}$ is a finite subset of $\mathcal{U}_{n}$, and $\bigcup_{n<\infty} \mathcal{J}_{n}$ is an open cover of $X$. It follows that in the generic extension, $X$ has the Menger property. Since this holds for all $\mathbb{B}(\kappa)$-generic filters, $1 \Rightarrow 2$ is proven.

Next we take up preservation and nonpreservation of the Hurewicz property under forcing with $\mathbb{B}(\kappa)$.

Theorem 43. Let $X$ be a topological space. If

$$
\mathbf{1}_{\mathbb{B}(\kappa)} \|- \text { " } \check{X} \text { has the Hurewicz property", }
$$

then $X$ has the Hurewicz property.

Proof. The proof of Theorem 37 adapts to this case.

Unlike the case of Cohen reals forcing, for $T_{1}$ regular Lindelöf spaces the Hurewicz property is preserved by random real forcing. In preparation for proving this, note first that regular Lindelöf spaces are normal, thus completely regular. Since such a space is $T_{1}$, it embeds as a subspace of $[0,1]^{\kappa}$ for some large enough cardinal $\kappa$. Since homeomorphisms preserve both the Hurewicz and the non-Hurewicz properties, we may restrict attention to subsets of $[0,1]^{\kappa}$. First, we prove the result for $\kappa=\omega$, the case of separable metric spaces. Then we return to the more general case. For the special case of a separable metric space we recall Proposition 38 and for the more general case recall Lemma 39.

TheOREM 44. Let $\kappa$ be an uncountable cardinal number. If $X$ is a $\mathrm{T}_{3}$ Hurewicz space then

$$
\mathbf{1}_{\mathbb{B}(\kappa)} \|- \text { "X has the Hurewicz property". }
$$

Proof. First we treat the case of subspaces of the Hilbert cube $\mathbb{H}$. Thus, assume $X \subseteq \mathbb{H}$ has the Hurewicz property. Assume that

$$
\mathbf{1}_{\mathbb{B}(\kappa)} \|-" \dot{G} \subseteq \dot{\mathbb{H}} \text { is a } \mathrm{G}_{\delta} \text {-set and } \check{X} \subseteq \dot{G} " .
$$

Fix $\mathbb{B}(\kappa)$-names $\dot{G}_{n}, n<\omega$, such that

$$
\mathbf{1}_{\mathbb{B}(\kappa)} \|-"(\forall n)\left(\dot{G}_{n} \supseteq \dot{G}_{n+1} \supseteq \dot{G} \text { are open and } \dot{G}=\bigcap_{n<\omega} \dot{G}_{n}\right) \text { ". }
$$


We may assume that for each $n$ we have $\mathbb{B}(\kappa)$-names $\dot{C}_{n}$ such that

$$
\mathbf{1}_{\mathbb{B}(\kappa)} \|-"(\forall n)\left(\dot{C}_{n}=\dot{\mathbb{H}} \backslash \dot{G}_{n}\right. \text {, a nonempty compact set)". }
$$

Let an $\epsilon>0$ be given. Consider a fixed $n$. For each $x \in X$ we have $\mathbf{1}_{\mathbb{B}(\kappa)} \|-" \check{x} \notin \dot{C}_{n} "$. For each $x$ choose a basic neighborhood $U_{n}(x)$ and a $\mathbb{B}(\kappa)$-name $\dot{V}_{n, x}$ so that

$$
\mu\left(\| \dot{C}_{n} \subseteq \dot{V}_{n, \check{x}} \text { open, and } \check{U}_{n}(\check{x}) \cap \dot{V}_{n, \check{x}}=\emptyset \|\right) \geq 1-\epsilon / 2^{n} .
$$

Now $\mathcal{U}_{n}=\left\{U_{n}(x): x \in X\right\}$ is an open cover of $X$.

Applying the fact that $X$ is Hurewicz, choose for each $n$ a finite set $\mathcal{F}_{n} \subseteq \mathcal{U}_{n}$ such that for each $x \in X$, for all but finitely many $n, x \in \bigcup \mathcal{F}_{n}$. For each $x \in X$ choose an $N(x) \in \mathbb{N}$ such that for all $n \geq N(x)$ we have $x \in \bigcup \mathcal{F}_{n}$.

For each $n$ let $I_{n}$ be a finite set for which $\left(U_{n}\left(x_{i}\right): i \in I_{n}\right)$ is a bijective enumeration of $\mathcal{F}_{n}$. Then for each $n$ define a $\mathbb{B}(\kappa)$-name $\dot{V}_{n}$ so that $\mathbf{1}_{\mathbb{B}(\kappa)} \|-$ " $\dot{V}_{n}=\bigcap_{i \in \check{I}_{n}} \dot{V}_{n, \check{x}_{i}}$ ". For each $n$ we have $\mu\left(\left\|\left(\bigcup \check{\mathcal{F}}_{n}\right) \cap \dot{V}_{n}=\emptyset\right\|\right) \geq 1-\epsilon / 2^{n}$.

For fixed $N$ define $X_{N}=\{x \in X: N(x)=N\}$. Then, for all $n \geq N$, $X_{N} \subseteq \cup \mathcal{F}_{n}$, and so, for each $n \geq N, \mu\left(\left\|\check{X}_{N} \cap \dot{V}_{n}=\emptyset\right\|\right) \geq 1-\epsilon / 2^{n}$ and consequently $\mu\left(\left\|\check{X}_{N} \cap \bigcup_{n \geq N} \dot{V}_{n}=\emptyset\right\|\right) \geq 1-\sum_{n=N}^{\infty} \epsilon / 2^{n}=1-\epsilon / 2^{N-1}$. This gives $\mu\left(\left\|\check{X}_{N} \subseteq \dot{\mathbb{H}} \backslash \bigcup_{n \geq N} \dot{V}_{n}\right\|\right) \geq 1-\epsilon / 2^{N-1}$.

Consequently,

$$
\mu\left(\left\|\check{X}_{N} \subseteq \dot{\mathbb{H}} \backslash\left(\left(\bigcup_{n \geq N} \dot{V}_{n}\right) \cap\left(\bigcap_{n<N} \dot{G}_{n}\right)\right) \subseteq \dot{G}\right\|\right) \geq 1-\epsilon / 2^{N-1} .
$$

But then, since $\| \dot{\mathbb{H}} \backslash \bigcup_{n \geq N} \dot{V}_{n}$ is $\sigma$-compact and $\bigcap_{n<N} \dot{G}_{n}$ is $\sigma$-compact $\|=$ $\mathbf{1}_{\mathbb{B}(\kappa)}$, we find that there is a $\mathbb{B}(\kappa)$-name $\dot{F}_{N}$ such that

$$
\mu\left(\| \dot{F}_{N} \text { is } \sigma \text {-compact and } \check{X}_{N} \subseteq \dot{F}_{N} \subseteq \dot{G} \|\right) \geq 1-\epsilon / 2^{N-1} .
$$

Since we also have $\mu\left(\left\|\check{X} \subseteq \bigcup_{n=1}^{\infty} \dot{F}_{n}\right\|\right) \geq 1-\epsilon \cdot\left(\sum_{n=1}^{\infty} 1 / 2^{n-1}\right)=1-2 \cdot \epsilon$, we conclude that $\mu(\|(\exists$ a $\sigma$-compact set $\dot{F})(\check{X} \subseteq \dot{F} \subseteq \dot{G}) \|) \geq 1-2 \cdot \epsilon$. As $\epsilon>0$ was arbitrary it follows that

$$
\mathbf{1}_{\mathbb{B}(\kappa)} \|-"\left(\forall G_{\delta} \text {-set } \dot{G} \subseteq \dot{\mathbb{H}}\right)\left(\exists \text { an } \mathrm{F}_{\sigma^{-}} \text {set } \dot{F}\right)(\check{X} \subseteq \dot{F} \subseteq \dot{G}) " .
$$

By Proposition 38 we have $\mathbf{1}_{\mathbb{B}(\kappa)} \|-$ " $\check{X}$ is Hurewicz".

Next we consider the general case. Let $X$ be a $T_{3}$ Hurewicz space and let $\lambda$ be the least infinite cardinal such that $X$ is homeomorphic to a subspace of $[0,1]^{\lambda}$. We may assume that $\lambda$ is uncountable. Write $\mathbb{H}_{\lambda}$ for $[0,1]^{\lambda}$.

Assume that $\mathbf{1}_{\mathbb{B}(\kappa)} \|-" \dot{G} \subseteq \mathbb{H}_{\lambda}$ is a $\mathrm{G}_{\delta^{-} \text {set }}$ and $\check{X} \subseteq \dot{G} \subseteq \dot{\mathbb{H}}_{\lambda}$ ". Since the random real forcing notion preserves the Lindelöf property, we have $\mathbf{1}_{\mathbb{B}(\kappa)} \|-$ " $\check{X}$ is Lindelöf". Thus, by Lemma 39 , we may assume $\dot{G}$ is such 
that there is a name $\dot{C}$ such that $\mathbf{1}_{\mathbb{B}(\kappa)}$ forces the statement

" $\dot{C}$ is a countable subset of $\check{\lambda}$ and $\dot{G}$ is homeomorphic to $\Pi_{\dot{C}}[\dot{G}] \times[0,1]^{\check{\lambda} \backslash \dot{C}}$ ". Since $\mathbb{B}(\kappa)$ has the countable chain condition, choose a countable set $C \subseteq \lambda$ such that $\mathbf{1}_{\mathbb{B}(\kappa)} \|-" \dot{C} \subseteq \check{C} "$. Then

$$
\mathbf{1}_{\mathbb{B}(\kappa)} \|-" \dot{G} \text { is homeomorphic to } \Pi_{\check{C}}[\dot{G}] \times[0,1]^{\check{\lambda} \backslash \check{C}} " .
$$

Since in the ground model $\Pi_{C}[X]$ is Hurewicz the first part implies $\mathbf{1}_{\mathbb{B}(\kappa)} \|-" \Pi_{\check{C}}[\check{X}] \subseteq \Pi_{\check{C}}[\dot{G}]$ is a $\mathrm{G}_{\delta}$-subset of $[0,1]^{\check{C}}$ and $\Pi_{\check{C}}[\check{X}]$ is Hurewicz".

Choose a $\mathbb{B}(\kappa)$-name $\dot{F}$ such that

$$
\mathbf{1}_{\mathbb{B}(\kappa)} \|-" \dot{F} \subseteq \dot{\mathbb{H}} \check{C} \text { is an } \mathrm{F}_{\sigma} \text {-set and } \Pi_{\check{C}}[\check{X}] \subseteq \dot{F} \subseteq \Pi_{\check{C}}[\dot{G}] " .
$$

But then applying the inverses of the projection maps we find

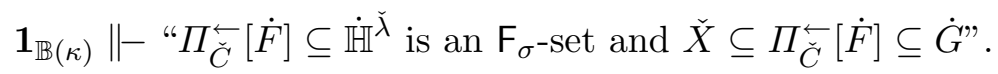

It follows that $\mathbf{1}_{\mathbb{B}(\kappa)} \|-$ " $\check{X}$ is a Hurewicz space".

Forcing with countably closed partially ordered sets. The Tikhonov power $\{0,1\}^{\omega_{1}}$ of the two-point discrete space is compact and thus has the Hurewicz (and thus Menger) property. But it is not indestructibly Lindelöf, and so unlike the Rothberger property (Theorem 21), the Hurewicz and Menger properties are not preserved by countably closed forcing. Juhász's spaces (Section 4, Example 4) are Hurewicz spaces but not indestructibly Lindelöf and so give points $\mathrm{G}_{\delta}$ examples of this fact. Call a space indestructibly Hurewicz (respectively indestructibly Menger) if in any generic extension by a countably closed partially ordered set the space is still Hurewicz (respectively Menger). It is clear that indestructibly Hurewicz implies indestructibly Menger, which implies indestructibly Lindelöf. The converse implications are false. However virtually the same proof as that of Theorem 21 gives:

TheOREM 45. The following are equivalent for a space $X$ :

1. $X$ is indestructibly Hurewicz.

2. $X$ has the Hurewicz property and is indestructibly Lindelöf.

THEOREM 46. The following are equivalent for a space $X$ :

1. $X$ is indestructibly Menger.

2. $X$ has the Menger property and is indestructibly Lindelöf.

When Lindelöf implies Gerlits-Nagy. Gerlits and Nagy also introduced the notion of a $\gamma$-space in [18]. According to [18] an open cover $\mathcal{U}$ of a space $X$ is an $\omega$-cover if $X \notin \mathcal{U}$, but for each finite set $F \subseteq X$ there is a $U \in \mathcal{U}$ such that $F \subseteq U$. The symbol $\Omega$ denotes the collection of (open) 
$\omega$-covers of a space. Call an open cover $\mathcal{U}$ of a space a $\gamma$-cover if it is infinite, and each infinite subset of $\mathcal{U}$ is still a cover of $X$. Let $\Gamma$ denote the collection of (open) $\gamma$-covers of a space.

Then a space is a $\gamma$-space if the selection principle $S_{1}^{\omega}(\Omega, \Gamma)$ holds: For each sequence $\left(\mathcal{U}_{n}: n \in \mathbb{N}\right)$ there is a sequence $\left(U_{n}: n \in \mathbb{N}\right)$ such that for each $n, U_{n} \in \mathcal{U}_{n}$, and each $x \in \mathbb{G}$ is in all but finitely many $U_{n}$ 's. Each $\gamma$-space is a Gerlits-Nagy space.

A topological space is a $P$-space if each countable intersection of open sets is still an open set. According to [18 the implication $1 \Rightarrow 4$ in Theorem 47 is due to F. Galvin. Since the remainder of these implications are trivial, we attribute this theorem to Galvin:

Theorem 47 (Galvin). Let $X$ be a P-space. The following are equivalent:

1. $X$ is Lindelöf.

2. $X$ is a Rothberger space.

3. $X$ is a Gerlits-Nagy space.

4. $X$ is a $\gamma$-space.

Proof. It is clear that $4 \Rightarrow 3 \Rightarrow 2 \Rightarrow 1$. For the implication $1 \Rightarrow 4$, see the Lemma preceding Theorem 3 of [18].

In particular, Lindelöf P-spaces are indestructibly Lindelöf.

L. Zdomskyy [60, Theorem 5] proved that it is consistent, relative to the consistency of ZFC, that for regular Lindelöf spaces the Rothberger property is equivalent to the Gerlits-Nagy property $\left(\left(^{7}\right)\right.$. The Continuum Hypothesis implies the existence of a Lusin set of real numbers. Lusin sets do not have the Gerlits-Nagy property, but have the Rothberger property. Among general topological spaces Lusin spaces need not be Rothberger spaces. This can be seen by considering a Sierpiński set of reals in the density topology. But Sierpiński sets have the Menger property in the density topology and, depending on axioms, may have the Hurewicz property in the density topology. For more on these matters see [2] and [43].

Gerlits-Nagy spaces and products. Next we examine products in the class of Gerlits-Nagy spaces. Corollary 48 solves Problem 6.6 of [56] and Problems 3.1 through 3.3 of Samet and Tsaban, listed in Section 3 of [55]:

$\left({ }^{7}\right)$ Zdomskyy shows that $\mathfrak{u}<\mathfrak{g}$ implies that regular Rothberger spaces have the Hurewicz property. Zdomskyy states that the blanket assumption for the paragraph containing Theorem 5's proof is that the spaces are hereditarily Lindelöf. This hypothesis is not used in proving Theorem 5, but is used in applying the result of Theorem 5 to an additivity problem. Zdomskyy states his results for paracompact Lindelöf spaces. It is well-known that regular Lindelöf spaces are paracompact. 
COROLlaRY 48. It is consistent that there is a set $X$ of real numbers such that $X$ has the Gerlits-Nagy property while $X^{2}$ does not, and yet all finite powers of $X$ have the Rothberger property.

Proof. Start with a model of $\mathrm{CH}$. Take a Sierpiński set $X$ such that $X \times X$ does not have the Menger property (see for example the remark following Theorem 2.1 of [27]). Sierpiński sets are strongly Hurewicz (see e.g. [44]). Since $X$ is a separable metric space it has the Lindelöf property in all finite powers.

Now add $\omega_{1}$ Cohen reals. By Theorem 11, in the generic extension $X$ has the Rothberger property in all finite powers (thus the Menger property in all finite powers). By Theorem 40, in the generic extension $X$ has the Hurewicz property and thus the Gerlits-Nagy property, but by Theorem 37 $X^{2}$ does not have the Hurewicz property, and thus not the Gerlits-Nagy property.

4. Examples. The following diagram indicates the relationships among the main classes of Lindelöf spaces considered in this paper:

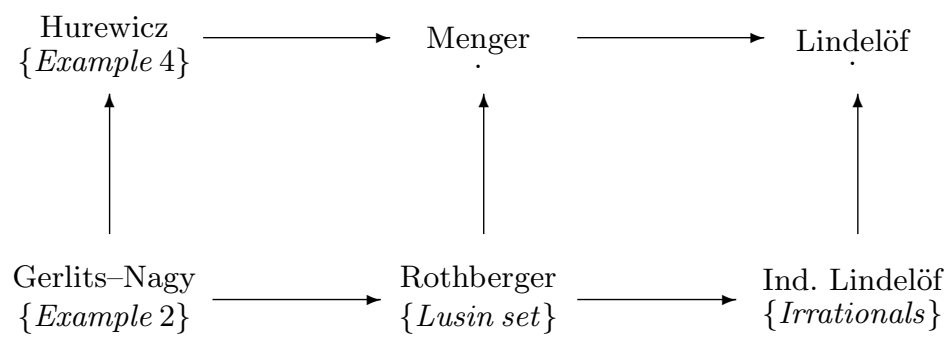

Fig. 1. Classes of points $\mathrm{G}_{\delta}$ Lindelöf spaces

In Figure 1 we depict the relationship among six classes of uncountable Lindelöf spaces with points $\mathrm{G}_{\delta}$. The spaces in the bottom row are indestructibly Lindelöf, while the spaces in the top row are not. Example 2 demonstrates that in ZFC the class in the bottom left is nonempty. Example 4 demonstrates that in ZFC the class of Hurewicz spaces is not included in the class of indestructibly Lindelöf spaces.

Except for the Rothberger and Gerlits-Nagy classes, the remaining classes are all distinct in ZFC: since the example in the top left corner is not a space as in the bottom right corner, no implication to the top row is an equivalence. Since the space of irrationals is not Menger, no implication from the middle column to the right column is an equivalence. It is consistent that also no implication from the first column is an equivalence: since a Lusin set is not Hurewicz, no implication from the first column to the middle one is an equivalence. For regular Lindelöf spaces it is independent 
whether the Rothberger and Gerlits-Nagy classes are distinct. It is true in ZFC that Menger does not imply Hurewicz.

EXAMPLE 1 (One-point compactification of an uncountable discrete space). Let $X$ be an uncountable discrete space and let $X^{*}$ denote its one-point compactification. Let $\infty$ be the additional point. Then $X^{*}$ is a Rothberger space but the point $\infty$ is not a $\mathrm{G}_{\delta}$. Being compact, $X^{*}$ is also a Hurewicz space, and thus a Gerlits-Nagy space.

EXAMPLE 2 (Moore's L-space is hereditarily Rothberger and hereditarily Hurewicz). In [35] Moore solved the famous L-space problem by constructing a non-separable zero-dimensional hereditarily Lindelöf subspace $X$ of the $\omega_{1}$-power of the unit circle. This space has among others the following properties:

1. $|X|=\aleph_{1}$.

2. $X$ is non-separable.

3. $X^{2}$ is not Lindelöf.

4. $X$ remains a hereditarily Lindelöf space in c.c.c. generic extensions.

5. Every continuous metrizable image of any subspace of $X$ is countable.

Item 5 implies that $X$ is hereditarily Rothberger: consider a subspace $Y$ of $X$, which we may assume is infinite. Let $\left(\mathcal{U}_{n}: n<\infty\right)$ be a sequence of open covers of $Y$. Since $Y$ is zero-dimensional and Lindelöf we may assume each $\mathcal{U}_{n}$ is countable and disjoint and enumerate it as $\left(U_{k}^{n}: k<\infty\right)$. Define a function $F: Y \rightarrow{ }^{\omega} \omega$ so that for $y \in Y$ we have: $F(y)(m)=k$ if, and only if, $y \in U_{k}^{m}$. Then $F$ is continuous and so by item $5, F[Y]$ is a countable subset of ${ }^{\omega} \omega$. Choose a $g \in{ }^{\omega} \omega$ such that for each $y \in Y$ the set $\{n: F(y)(n)=g(n)\}$ is infinite. Then $\left(U_{g(n)}^{n}: n<\infty\right)$ witnesses the Rothberger property of $Y$ for the sequence $\left(\mathcal{U}_{n}: n<\infty\right)$.

Since countable subsets of ${ }^{\omega} \omega$ are bounded, we also see that $X$ has the Hurewicz property hereditarily. Thus, $X$ is hereditarily a Gerlits-Nagy space.

It follows from item 3 that $X$ is a ZFC example of the total failure of preservation of selection properties under finite powers. Since the space is a Lindelöf subspace of a power of a Tikhonov space, it is $\mathrm{T}_{4}$. And then, since it is hereditarily Lindelöf, its points are $\mathrm{G}_{\delta}$ : Consider an $x \in X$. For each $y \in X \backslash\{x\}$ choose an open neighborhood $U_{y}$ whose closure does not contain $x$. Then $\left\{U_{y}: y \in X \backslash\{x\}\right\}$ is an open cover of $X$. Since $X \backslash\{x\}$ is Lindelöf, choose a countable subset covering $X \backslash\{x\}$. The complements of the closures of the sets in this countable cover witness that $x$ is a $\mathrm{G}_{\delta}$ point. Moore's spaces are also not first countable (following from item 4) but they are Fréchet. These spaces can also be realized as subspaces of $\left(\omega_{1} \mathbb{Z}, \mathcal{T}\right)$, where $\mathcal{T}$ is the usual product topology on $\omega_{1}$ copies of the discrete space of integers. 
Hereditarily Lindelöf spaces are indestructibly Lindelöf and thus remain Lindelöf in any countably closed forcing extension. Thus, any ground model subspace of a hereditarily Lindelöf space remains Lindelöf in every countably closed forcing extension. But in the forcing extension the space may acquire new subspaces that are not ground-model subspaces. We now show that even these new subspaces are Lindelöf.

Recall that a space is right-separated if it has a well-ordering such that each element of the space has a neighborhood that does not include any of the successors of that point. A hereditarily Lindelöf space has no uncountable right-separated subspace. If a space has an uncountable right-separated subspace, then it has one of the form $\left\{x_{\alpha}: \alpha<\omega_{1}\right\}$ where for each $\alpha$, $\left\{x_{\beta}: \beta<\alpha\right\}$ is open in $\left\{x_{\alpha}: \alpha<\omega_{1}\right\}$.

Lemma 49. Countably closed forcing preserves being a hereditarily Lindelöf space.

Proof. Suppose the contrary and let $L$ be a hereditarily Lindelöf space and let $\mathbb{Q}$ be a countably closed partially ordered set such that

$$
\mathbf{1} \|- \text { "L } \check{L} \text { is not hereditarily Lindelöf". }
$$

Choose for $\alpha<\omega_{1} \mathbb{Q}$-names $\dot{x}_{\alpha}$ and names for open sets $\dot{U}_{\alpha}$ such that

$$
1 \|- \text { " }\left\{\dot{x}_{\alpha}: \alpha<\omega_{1}\right\} \subseteq \check{L} \text { is right-separated by } \dot{U}_{\alpha}, \alpha<\omega_{1} \text { ". }
$$

Recursively construct $p_{\alpha}, y_{\alpha}$ and $V_{\alpha}$ in $V$ so that

1. $\alpha<\beta<\omega_{1} \Rightarrow p_{\beta} \leq p_{\alpha}$;

2. $\alpha<\omega_{1} \Rightarrow y_{\alpha} \in V_{\alpha}$

3. $\alpha<\omega_{1} \Rightarrow p_{\alpha} \|-" \dot{x}_{\alpha}=\check{y}_{\alpha} \in \check{V}_{\alpha} \subseteq \dot{U}_{\alpha}$ ".

This is done as follows: Suppose $p_{\alpha}$ as well as $y_{\alpha} \in L$ and open subsets $V_{\alpha}$ of $L$ are given, such that 1,2 and 3 hold for $\alpha<\beta$.

Take $q$ below all $p_{\alpha}$ and select $y_{\beta}$ and an open set $V_{\beta}$ and extension $p_{\beta}$ of $q$, for which $p_{\beta} \|-{ }^{\prime} \dot{x}_{\beta}=\check{y}_{\beta} \in \check{V}_{\beta} \subseteq \dot{U}_{\beta}$ ". This can be done since the ground-model open sets form a base for the open sets in the extension.

But then $Y=\left\{y_{\beta}: \beta<\omega_{1}\right\} \subseteq L$ is right-separated in the ground model. This is witnessed by the $V_{\alpha}$ 's. For suppose $\beta>\alpha$ and $y_{\beta} \in V_{\alpha}$. Then

$$
p_{\beta} \|-" \dot{x}_{\beta}=\check{y}_{\beta} \text { and } \check{y}_{\beta} \in \check{V}_{\alpha} \subseteq \dot{U}_{\alpha} ",
$$

so $p_{\beta} \|-" \dot{x}_{\beta} \in \dot{U}_{\alpha}$ ", a contradiction.

EXAMPLE 3 (Gorelic's example). Gorelic's space, $\mathbb{G}$, is obtained by countably closed forcing $\mathbb{P}$. An uncountable cardinal number $\kappa$ with $\kappa^{\aleph_{1}}=\kappa$ is taken, and then a subspace $\mathbb{G} \subseteq{ }^{\kappa}\{0,1\}$ of cardinality $\kappa$ is obtained in a generic extension which has $2^{\aleph_{1}}=\kappa$, such that $\mathbb{G}$ is zero-dimensional, Lindelöf, and has each point $\mathrm{G}_{\delta}$. 
Indeed, $\mathbb{G}$ has both the Rothberger property and the Hurewicz property, as we shall now show. Towards this we first describe Gorelic's construction. First, choose a partition of $\kappa$ into pairwise disjoint countable sets $A_{\alpha}$, $\alpha<\kappa$. For each $\beta<\kappa$ choose a countable dense subset $H_{\beta}$ of ${ }_{\beta}\{0,1\}$ and enumerate it as $\left\{h_{\alpha}^{\beta}: \alpha \in A_{\beta}\right\}$.

The elements of $\mathbb{P}$ are pairs $(F, T)$ where $F$ and $T$ are as follows:

- Both $F$ and $T$ are countable sets.

- There is a countable set $C \subseteq \kappa$ such that each element of $F$ is a function with domain $D=\bigcup_{\beta \in C} A_{\beta}$.

- There is a countable set $G=\left\{g_{\alpha}: \alpha \in A\right\}$ with each $g_{\alpha}$ a function from $D \backslash A_{\alpha}$ to $\{0,1\}$.

- $F$ can be indexed as $\left\{f_{\xi}: \xi \in D\right\}$ such that whenever $\beta \in C$ and $\alpha \in A_{\beta}$ then $f_{\alpha}\left\lceil_{A_{\beta}}=h_{\alpha}^{\beta}\right.$ and $f_{\alpha}\left\lceil D \backslash A_{\beta}=g_{\beta}\right.$.

- For each $\alpha \in C$ and each $\beta \in C \backslash\{\alpha\}, g_{\beta}\left\lceil A_{\alpha} \neq h_{\xi}^{\alpha}\right.$ for each $\xi \in A_{\alpha}$.

- Each element of $T$ is a set $B \subseteq \operatorname{Fn}(D, 2)$, with $\left\{[\sigma] \subseteq{ }^{A}\{0,1\}: \sigma \in B\right\}$ a cover of $F$.

The order on $\mathbb{P}$ is defined as follows:

$$
q=\left(F_{2}, T_{2}\right) \leq\left(F_{1}, T_{1}\right)=p
$$

if, letting $C_{p}$ and $C_{q}$ be the associated $C^{\prime}$ 's, and $D_{p}$ and $D_{q}$ the associated $D$ 's, we have:

1. $C_{q} \supseteq C_{p}$.

2. For each $\alpha \in D_{p}, f_{\alpha}^{q}\left\lceil_{D_{p}}=f_{\alpha}^{p}\right.$.

3. $T_{q} \supseteq T_{p}$.

When $H$ is a $\mathbb{P}$-generic filter, put for each $\alpha \in \kappa$,

$$
f_{\alpha}=\bigcup\left\{f_{\alpha}^{p}: p \in H \text { and } \alpha \in D_{p}\right\} .
$$

Then put $\mathbb{G}=\left\{f_{\alpha}: \alpha \in \kappa\right\}$. Let $\Gamma$ be a $\mathbb{P}$-name for $\mathbb{G}$.

Here are some observations about $p=(F, T) \in \mathbb{P}$ (in the above notation):

1. If $U \in T$ then $p \|-$ " $(\forall f \in \Gamma)(\exists \sigma \in U)(\sigma \subseteq f)$ ".

2. If $\tau$ is a $\mathbb{P}$-name such that $p \|-$ " $\tau$ is a cover of $\Gamma$ by basic open sets" then there is a $q<p$ with a $B \in T_{q}$ such that $q \|-"\left\{[\sigma] \subseteq{ }^{\kappa}\{0,1\}\right.$ : $\sigma \in B\} \subseteq \tau$ covers $\Gamma^{\prime \prime}$.

3. For $B \subseteq \operatorname{Fn}\left(D_{p}, 2\right)$ such that $\left\{[\sigma] \subseteq D^{D}\{0,1\}: \sigma \in B\right\}$ covers $F \subseteq$ $D_{p}\{0,1\}$ put $q=(F, T \cup\{B\})$. Then $q \in \mathbb{P}$ and $q \leq p$.

By the first item above, $p$ forces that $\left\{[\sigma] \subseteq{ }^{\kappa}\{0,1\}: \sigma \in B\right\}$ is an open cover of $\mathbb{G}$ whenever $B \in T_{p}$.

To see that for each $p \in \mathbb{P}$ we have

$$
p \|- \text { " } \Gamma \text { has the Rothberger property" }
$$


consider $\mathbb{P}$-names $\tau_{n}$ such that

$$
p \|-"(\forall n)\left(\tau_{n} \text { is a basic open cover of } \Gamma\right) " .
$$

Now using the second remark above, recursively choose a sequence $p_{n}=$ $\left(F_{n}, T_{n}\right)$ and $B_{n}, n<\infty$, such that:

1. $B_{n} \in T_{n}$ and $p_{n} \|-$ " $\left\{[\sigma] \subseteq{ }^{\kappa}\{0,1\}: \sigma \in B_{n}\right\} \subseteq \tau_{n}$ covers $\Gamma$ ".

2. $p_{1}<p$ and for all $n, p_{n+1}<p_{n}$.

Put $D=\bigcup_{n<\infty} D_{p_{n}}$, a countable subset of $\kappa$, and for $\alpha \in D$ put $f_{\alpha}=$ $\bigcup\left\{f_{\alpha}^{p_{n}}: \alpha \in D_{p_{n}}\right\}$. Put $F=\left\{f_{\alpha}: \alpha \in D\right\}$ and $T=\bigcup\left\{T_{n}: n<\infty\right\}$. Then $q=(F, T)$ is an element of $\mathbb{P}$ and for all $n, q \leq p_{n}$.

Now $F \subseteq{ }^{D}\{0,1\}$ is countable, and for each $n, \mathcal{U}_{n}=\left\{[\sigma] \subseteq{ }^{D}\{0,1\}\right.$ : $\left.\sigma \in B_{n}\right\}$ is an open cover of $F$. Since $F$ is countable we can choose for each $n$ a $\sigma_{n} \in B_{n}$ such that $\left\{\left[\sigma_{n}\right] \subseteq D\{0,1\}: n<\infty\right\}$ is an open cover of $F$. Note that $B=\left\{\sigma_{n}: n<\infty\right\} \subseteq \operatorname{Fn}(D, 2)$. But then we have

$$
p_{\omega}=(F, T \cup\{B\}) \in \mathbb{P} \text {. }
$$

Then $B$ witnesses $\left({ }^{8}\right)$ that

$$
p_{\omega} \|-"(\forall n)\left(\exists U_{n} \in \tau_{n}\right)\left(\left\{U_{n}: n<\infty\right\} \text { covers } \Gamma\right) " .
$$

We have shown that the set of conditions forcing that $\mathbb{G}$ has the Rothberger property is dense below any element of $\mathbb{P}$.

To show that $\mathbb{G}$ has the Hurewicz property, instead of choosing singletons from each $B_{n}$ choose finite sets such that each element of $F$ is covered by all but finitely many of the unions of these finite sets, and let the $B$ in the definition of $p_{\omega}$ be the union of these finite sets.

With a little bit more work one can show that $\mathbb{G}$ is a $\gamma$-space. It follows that $\mathbb{G}$ is a $\gamma$-space with points $G_{\delta}$ and yet has cardinality $2^{\aleph_{1}}>\aleph_{1}=2^{\aleph_{0}}$. The fact that $\mathbb{G}$ has all points $G_{\delta}$ is explained in Gorelic's original article: see point (5) and the subsequent remark on page 604 of [19].

ExAmple 4 (Juhász's examples). In [26, Example 7.2], Juhász gave examples of Lindelöf spaces with points $\mathrm{G}_{\delta}$ which are of arbitrarily large cardinality below the first measurable cardinal. We give, for the reader's convenience, the description from [51] for these.

Consider an infinite cardinal $\kappa$ less than the first measurable cardinal. Recursively define

$$
\kappa_{0}=\kappa, \quad \kappa_{n+1}=2^{\kappa_{n}}, \quad \kappa_{\omega}=\sup \left\{\kappa_{n}: n<\infty\right\} .
$$

For an infinite set $S$, let $\mathcal{F}(S)$ denote the set of nonprincipal ultrafilters on $S$. For $A \subseteq S$ put $\hat{A}=\{\mathcal{U} \in \mathcal{F}(S): A \in \mathcal{U}\}$. And for $\mathcal{U} \in \mathcal{F}(\mathcal{F}(S))$ put $\mathcal{U}^{\prime}=\{A \subseteq S: \hat{A} \in \mathcal{U}\}$. Then $\mathcal{U}^{\prime}$ is an ultrafilter and a member of $\mathcal{F}(S)$.

$\left(^{8}\right)$ Define the $\mathbb{P}$-name $\mu=\left\{\left(\left\langle n, \sigma_{n}\right\rangle, \mathbf{1}\right): n<\infty\right\}$. Then check that $p_{\omega}$ forces that $\mu$ codes a sequence of such $U_{n}$ 's. 
Define $J_{0}=\kappa$ and for each $n$ define $J_{n+1}=\mathcal{F}\left(J_{n}\right)$. Finally, put $\mathbb{J}_{\kappa}=$ $\bigcup_{n<\infty} J_{n}$.

Now for each $n$, and for $\mathcal{U} \in X_{n+1}$ define $\mathcal{U}^{(i)}$ for $i \leq n$ as follows:

$$
\mathcal{U}^{(0)}=\mathcal{U}, \quad \mathcal{U}^{(i+1)}=\left(\mathcal{U}^{(i)}\right)^{\prime} .
$$

The topology on $\mathbb{J}_{\kappa}$ is defined by describing neighborhood bases for points:

- Points of $J_{0}$ are isolated.

- For $\mathcal{U} \in J_{n+1}$, all sets of the form

$$
\{\mathcal{U}\} \cup \bigcup_{i=0}^{n} A^{(i)}, \quad A^{(i)} \in \mathcal{U}^{(i)}, 0 \leq i \leq n,
$$

constitute a neighborhood base of $\mathcal{U}$.

These spaces are not $T_{2}$. Juhász showed in [26] that they have each point $\mathrm{G}_{\delta}$. Tall showed in [51, Theorem 19] that they are destructibly Lindelöf. Thus, they are not Rothberger.

The following crucial observation of Juhász provides a tool to show that spaces of the form $\mathbb{J}_{\kappa}$ are Hurewicz:

Lemma 50 (Juhász). For any set $S$ and function $f: \mathcal{F}(S) \rightarrow \mathcal{P}(S)$ such that for each $\mathcal{U} \in \mathcal{F}(S)$ we have $f(\mathcal{U}) \in \mathcal{U}$, there are finitely many ultrafilters $\mathcal{U}_{1}, \ldots, \mathcal{U}_{n}$ in $\mathcal{F}(S)$ such that $\left|S \backslash \bigcup_{1 \leq i \leq n} f\left(\mathcal{U}_{i}\right)\right|<\aleph_{0}$.

Lemma 50 implies for each $n$ that from each open cover of $J_{n+1}$ one can choose finitely many members whose union covers all but finitely many points of $J_{n}$.

We can show that the property of this lemma is preserved in the random real extension, and thus $\mathbb{J}_{\kappa}$ remains a Hurewicz space in the random real extension. We do not know if this is also the case with Cohen reals.

Lemma 51. For each infinite cardinal $\kappa$ the space $\mathbb{J}_{\kappa}$ has the Hurewicz property.

Proof. Apply Lemma 50: Given a sequence $\left(\mathcal{G}_{n}: n<\infty\right)$ of open covers of $\mathbb{J}_{\kappa}$, choose for each $n$ a finite set $\mathcal{H}_{n} \subseteq \mathcal{G}_{n}$ which covers $\bigcup_{i \leq n} J_{i}$. It follows that each element of $J_{\omega}$ is contained in all but finitely many $\bigcup \mathcal{H}_{n}$.

Another interesting property of these spaces is given in Theorem 21 of [5]:

THEOREM 52 (Baumgartner-Tall). $\mathbb{J}_{\kappa}$ does not have a Lindelöf subspace of cardinality $\aleph_{1}$.

Consider $\mathbb{J}_{\kappa}$ from the ground model in a generic extension by $\mathbb{P}\left(\aleph_{1}\right)$, the partially ordered set for adding $\aleph_{1}$ Cohen reals. By Theorem 11, $\mathbb{J}_{\kappa}$ acquired the Rothberger property. Now recall Lemma 25 of [5]: 
Lemma 53 (Baumgartner-Tall). Suppose $X$ is a space in the ground model. Force with a property $K$ partial order. Then if $X$ has a Lindelöf subspace of size $\aleph_{1}$ in the extension, it has one in the ground model.

Both the Cohen reals partial order $\mathbb{P}(\kappa)$ and the random reals partial order $\mathbb{B}(\kappa)$ have property $\mathrm{K}$.

It follows that $\mathbb{J}_{\kappa}$ still does not have a Lindelöf subspace of cardinality $\aleph_{1}$ in the generic extension. Thus, it is consistent relative to the consistency of ZFC that there are spaces of arbitrarily large cardinality below the first measurable cardinal which have points $\mathrm{G}_{\delta}$ and the Rothberger property, and no Lindelöf subspace of cardinality $\aleph_{1}$

Examining Corollary 24 of [5] we see that it is consistent that there is a $T_{1}$ Rothberger space of cardinality $\aleph_{2}=\left(2^{\aleph_{0}}\right)^{+}$which has no Lindelöf subspace of cardinality $\aleph_{1}$. Indeed: if we take a $\mathbb{J}_{\kappa}$, add $\aleph_{1}$ Cohen reals to make it a Rothberger space, and then collapse its cardinality to $\aleph_{2}$ with conditions of size $\leq \aleph_{1}$, we obtain a Rothberger space with no Lindelöf subspace of size $\aleph_{1}$.

Example 5 (Koszmider and Tall's example). Juhász's spaces are not $\mathrm{T}_{2}$ but have points $\mathrm{G}_{\delta}$. In [31] Koszmider and Tall constructed by countably closed forcing $\mathbb{P}$ over a model of $\mathrm{CH}$ a subspace $\mathbb{K}$ of $\left(\omega_{2} 2, \mathcal{T}_{2}\right)$, where $\mathcal{T}_{2}$ denotes the refinement of the usual topology on ${ }^{\omega_{2}} 2$ obtained by declaring all $G_{\delta}$-sets from the usual topology open. Thus $\mathbb{K}$ is a $\mathrm{P}$-space which is $\mathrm{T}_{3}$ and Lindelöf (thus $\mathrm{T}_{4}$ ), is of cardinality $\aleph_{2}$, and it has no Lindelöf subspace of cardinality $\aleph_{1}$. It follows from Theorem 47 that $\mathbb{K}$ has the Gerlits-Nagy property and yet fails to have a Lindelöf subspace of cardinality $\aleph_{1}$.

Example 6 (Stationary Aronszajn lines). In 54 Todorčević investigates the status of certain selection principles on the class of Aronszajn lines. These are first countable spaces, and in Theorem 1 of [54] it is shown that stationary Aronszajn lines are Rothberger spaces. Theorem 3 of [54], combined with a result of [7] that for each Lindelöf space $X$, if each continuous function from $X$ to ${ }^{\omega} \mathbb{R}$ is bounded in the eventual domination order, then the space $X$ is a Hurewicz space, shows these also have the Hurewicz property. Thus, the stationary Aronszajn lines have the Gerlits-Nagy property. In Theorem 6 of [54] Todorčević shows that the more restrictive class of stationary Countryman lines indeed are $\gamma$-spaces, and that though this property is preserved by finite powers, it is not preserved by finite unions, and not even the Lindelöf property is preserved by finite products of $\gamma$-spaces. Since the Rothberger property and the Hurewicz property are preserved by countable unions, these examples also demonstrate that the class of $\gamma$-spaces is more restrictive than the class of Gerlits-Nagy spaces. Each of the above mentioned examples can be taken to be a space of cardinality $\aleph_{1}$. 
Acknowledgements. We thank the referee for a very careful reading of the paper, and for pointing out that Cohen real forcing does not in general preserve the Hurewicz property. After this paper was completed Masaru Kada found an elegant description of a class of forcing posets which preserve the Rothberger property - see [28]. This class includes the Cohen reals posets, the random reals posets as well as the countably closed posets. Thus, Kada's results give a simultaneous generalization of three of our results on preserving the Rothberger property. We thank Kada for communicating his results to us.

Research of F. D. Tall was supported by Grant A-7354 of the Natural Sciences and Engineering Research Council of Canada.

\section{References}

[1] A. V. Arhangel'skii, On the cardinality of bicompacta satisfying the first axiom of countability, Soviet Math. Dokl. 10 (1969), 951-955.

[2] L. F. Aurichi and F. D. Tall, Lindelöf spaces which are indestructible, productive or D, Topology Appl., to appear.

[3] T. Banakh and L. Zdomskyy, Separation properties between the $\sigma$-compactness and Hurewicz property, Topology Appl. 156 (2008), 10-15.

[4] T. Bartoszyński and H. Judah, Borel images of sets of reals, Real Anal. Exchange 20 (1995), 1-23.

[5] J. E. Baumgartner and F. D. Tall, Reflecting Lindelöfness, Topology Appl. 122 (2002), 35-49.

[6] J. E. Baumgartner and E. van Douwen, Strong real-compactness and weakly measurable cardinals, ibid. 35 (1990), 239-251.

[7] M. Bonanzinga, F. Cammaroto and M. Matveev, Projective versions of selection principles, ibid. 157 (2010), 874-893.

[8] E. Borel, Sur la classification des ensembles de mesure nulle, Bull. Soc. Math. France 47 (1919), 97-125.

[9] T. Carlson, Extending Lebesgue measure by infinitely many sets, Pacific J. Math. 115 (1984), 33-45.

[10] —, Strong measure zero and strongly meager sets, Proc. Amer. Math. Soc. 118 (1993), 577-586.

[11] J. Chaber and R. Pol, A remark on Fremlin-Miller theorem concerning the Menger property and Michael concentrated sets, manuscript, 2002.

[12] P. Daniels and G. Gruenhage, The point-open type of subsets of the reals, Topology Appl. 37 (1990), 53-64.

[13] A. Dow, Two applications of reflection and forcing to topology, in: Z. Frolík (ed.), General Topology and Its Relations to Modern Analysis and Algebra VI, Proc. Sixth Prague Topology Symposium 1986, Helderman, Berlin, 1988, 155-172.

[14] A. Dow, F. D. Tall and W. A. R. Weiss, New proofs of the consistency of the normal Moore space conjecture, Topology Appl. 37 (1990), 33-51.

[15] - , - - New proofs of the consistency of the normal Moore space conjecture II, ibid. 37 (1990), 115-129.

[16] D. H. Fremlin and A. W. Miller, On some properties of Hurewicz, Menger and Rothberger, Fund. Math. 129 (1988), 17-33. 
[17] F. Galvin, Indeterminacy of point-open games, Bull. Acad. Polon. Sci. 26 (1978), 445-449.

[18] J. Gerlits and Zs. Nagy, Some properties of $C(X)$, I, Topology Appl. 14 (1982), $151-161$.

[19] I. Gorelic, The Baire Category and forcing large Lindelöf spaces with points $\mathrm{G}_{\delta}$, Proc. Amer. Math. Soc. 118 (1993), 603-607.

[20] R. Grunberg, L. R. Junqueira and F. D. Tall, Forcing and normality, Topology Appl. 84 (1998), 45-174.

[21] W. Hurewicz, Über eine Verallgemeinerung des Borelschen Theorems, Math. Z. 24 (1925), 401-425.

[22] —, Über Folgen stetiger Funktionen, Fund. Math. 9 (1927), 193-204.

[23] A. Iwasa, Covering properties and Cohen forcing, Topology Proc. 31 (2007), 553559.

[24] T. Jech, Set Theory, 2nd ed., Springer, 1997.

[25] H. Judah, S. Shelah and W. H. Woodin, The Borel Conjecture, Ann. Pure Appl. Logic 50 (1990), 255-269.

[26] I. Juhász, Cardinal Functions in Topology - Ten Years Later, Math. Centrum, Amsterdam, 1980.

[27] W. Just, A. W. Miller, M. Scheepers and P. J. Szeptycki, Combinatorics of open covers (II), Topology Appl. 73 (1996), 241-266.

[28] M. Kada, Preserving the Lindelöf property under forcing extensions, Topology Proc., to appear.

[29] R. W. Knight, A topological application of flat morasses, Fund. Math. 194 (2007), $45-66$.

[30] Lj. D. R. Kočinac and M. Scheepers, Combinatorics of open covers (VII): Groupability, ibid. 179 (2003), 131-155.

[31] P. Koszmider and F. D. Tall, A Lindelöf space with no Lindelöf subspace of size $\aleph_{1}$, Proc. Amer. Math. Soc. 130 (2002), 2777-2787.

[32] R. Laver, On the consistency of Borel's conjecture, Acta Math. 137 (1976), 151-169.

[33] A. Lévy and R. M. Solovay, Measurable cardinals and the Continuum Hypothesis, Israel J. Math. 5 (1967), 234-248.

[34] K. Menger, Einige Überdeckungssätze der Punktmengenlehre, Sitzungsberichte Abt. 2a, Mathematik, Astronomie, Physik, Meteorologie und Mechanik, Wiener Akademie, Wien 133 (1924), 421-444.

[35] J. T. Moore, A solution to the L-space problem, J. Amer. Math. Soc. 19 (2006), $717-736$.

[36] J. Nagata, On the countable sum of zero-dimensional spaces, Fund. Math. 48 (1960), $1-14$.

[37] A. Nowik, M. Scheepers and T. Weiss, The algebraic sum of sets of real numbers with strong measure zero, J. Symbolic Logic 63 (1998), 301-324.

[38] J. Pawlikowski, Undetermined sets of point-open games, Fund. Math. 144 (1994), 279-285.

[39] - Strongly meager sets and subsets of the plane, ibid. 156 (1998), 279-287.

[40] E. Pearl (ed.), Open Problems in Topology II, Elsevier, 2007.

[41] F. Rothberger, Eine Verschärfung der Eigenschaft C, Fund. Math. 30 (1938), 50-55.

[42] M. Scheepers, Combinatorics of open covers (I): Ramsey theory, Topology Appl. 69 (1996), 31-62.

[43] -, Selection principles and Sierpiński sets, Acta Math. Sinica 23 (2007), 1153-1162.

[44] M. Scheepers and B. Tsaban, The combinatorics of Borel covers, Topology Appl. 121 (2002), 357-382. 
[45] S. Shelah, On some problems in general topology, in: Contemp. Math. 192 (1996), 91-101.

[46] S. Shelah and L. J. Stanley, S-Forcing I, A "black-box" theorem for morasses, with applications to super Souslin trees, Israel J. Math. 43 (1982), 185-224.

[47] W. Sierpiński, Sur un ensemble non dénombrable, dont toute image continue est de mesure nulle, Fund. Math. 11 (1928), 301-304.

[48] Y. M. Smirnov, On universal spaces for certain classes of infinite-dimensional spaces, Amer. Math. Soc. Transl. (2) 21 (1962), 35-50.

[49] R. M. Solovay, Real-valued measurable cardinals, in: Axiomatic Set Theory, Proc. Sympos. Pure Math. 13, Amer. Math. Soc., 1971, 397-428.

[50] J. Steprāns, Combinatorial consequences of adding Cohen reals, in: Israel Math. Conf. Proc. 6, Bar-Ilan Univ., 1993, 583-617.

[51] F. D. Tall, On the cardinality of Lindelöf spaces with points $\mathrm{G}_{\delta}$, Topology Appl. 63 (1995), 21-38.

[52] —, Lindelöf spaces which are Menger, Hurewicz, Alster, productive or D, ibid., to appear.

[53] R. Telgársky, On games of Topsøe, Math. Scand. 54 (1984), 170-176.

[54] S. Todorčević, Aronszajn orderings, Publ. Inst. Math. 57 (71) (1995), 29-46.

[55] B. Tsaban, SPM Bulletin 18 (2006), p. 7 (http://arxiv.org/PS_cache/math/pdf/ 0609/0609401v1.pdf).

[56] -, Selection principles and special sets of reals, in [40, 91-108.

[57] B. Tsaban and L. Zdomskyy, Combinatorial images of sets of reals and semifilter trichotomy, J. Symbolic Logic 73 (2008), 1278-1288.

[58] —, - Scales, fields and a problem of Hurewicz, J. Eur. Math. Soc. 10 (2008), 837-866.

[59] D. Velleman, Morasses, diamond and forcing, Ann. Math. Logic 23 (1982), 199-281.

[60] L. Zdomskyy, A semifilter approach to selection principles, Comment. Math. Univ. Carolin. 46 (2005), 525-539.

Marion Scheepers

Department of Mathematics

Boise State University

1910 University Drive

Boise, ID 83725, U.S.A.

E-mail: mscheepe@boisestate.edu
Franklin D. Tall Department of Mathematics University of Toronto Toronto, Ontario M5S2E4, Canada E-mail: f.tall@utoronto.ca

Received 12 February 2009; in revised form 30 April 2010 